PALEO

Revue d'archéologie préhistorique

$18 \mid 2006$

Varia

\title{
Composition granulométrique des assemblages lithiques
}

Application à l'étude taphonomique des sites paléolithiques

Particle size distribution of lithic assemblages and taphonomy of paleolithic sites

Pascal Bertran, Émilie Claud, Luc Detrain, Arnaud Lenoble, Bertrand

Masson et Luc Vallin

\section{OpenEdition}

Journals

Édition électronique

URL : http://journals.openedition.org/paleo/111

DOI : 10.4000/paleo.111

ISSN : 2101-0420

Éditeur

SAMRA

Édition imprimée

Date de publication : 1 décembre 2006

Pagination : 7-36

ISSN : $1145-3370$

Référence électronique

Pascal Bertran, Émilie Claud, Luc Detrain, Arnaud Lenoble, Bertrand Masson et Luc Vallin,

"Composition granulométrique des assemblages lithiques », PALEO [En ligne], 18 | 2006, mis en ligne

le 23 avril 2009, consulté le 07 juillet 2020. URL : http://journals.openedition.org/paleo/111 ; DOI :

https://doi.org/10.4000/paleo.111

\section{(c) $(1) \Theta \Theta$}

PALEO est mis à disposition selon les termes de la licence Creative Commons Attribution - Pas d'Utilisation Commerciale - Pas de Modification 4.0 International 


\title{
COMPOSITION GRANULOMÉTRIQUE DES ASSEMBLAGES LITHIQUES, APPLICATION À L'ÉTUDE TAPHONOMIQUE DES SITES PALÉOLITHIQUES
}

\author{
Pascal BERTRAN ${ }^{(1,2)}$, Émilie CLAUD ${ }^{(2)}$, Luc DETRAIN ${ }^{(1,2)}$, \\ Arnaud LENOBLE ${ }^{(2)}$, Bertrand MASSON ${ }^{(3)}$ et Luc VALLIN ${ }^{(3)}$
}

\begin{abstract}
Résumé : Cet article propose une revue sur l'utilisation de la granulométrie du matériel archéologique dans le cadre de l'étude taphonomique des sites paléolithiques. La composition granulométrique numérale de la fraction supérieure à $2 \mathrm{~mm}$ produite par des débitages expérimentaux est très constante quel que soit le mode de taille mis en œuvre et obéit à une loi exponentielle décroissante. Des différences apparaissent néanmoins en fonction d'un certain nombre de facteurs, comme la matière première utilisée, la dimension des produits recherchés ou la maîtrise du tailleur. Ces différences sont cependant minimes et la variation qui affecte les proportions des différentes classes granulométriques reste inférieure à $15 \%$ d'un débitage ou d'un façonnage à l'autre. La composition d'assemblages lithiques archéologiques peut être comparée aux données expérimentales, qui donnent une bonne image de ce que devait être la granulométrie du matériel archéologique taillé sur le site. La mise en évidence d'un déficit ou, à l'inverse, d'une surreprésentation de certaines fractions témoigne d'un tri dont l'origine peut être anthropique ou naturelle. Les expériences concernant les tris granulométriques provoqués par des écoulements naturels (ruissellement, rivières) sont décrites. Les courbes granulométriques caractéristiques obtenues sont différentes selon que l'on se trouve dans une zone de résidualisation, de transit ou d'accumulation du matériel. Les tris naturels ont en commun de se marquer surtout dans la fraction la plus fine $(<5 \mathrm{~mm})$, contrairement aux tris d'origine anthropique. Plusieurs exemples d'études sur des sites paléolithiques français sont présentés et montrent les potentialités de cet outil pour comprendre les processus de formation des sites. De manière à minimiser les incertitudes qui pèsent encore sur l'interprétation de l'origine des tris granulométriques, un certain nombre de recommandations sur le choix des critères les plus robustes et sur l'insertion de ce type d'étude au sein de la démarche analytique générale d'un site est proposé.
\end{abstract}

Mots-clés : granulométrie, taphonomie, Paléolithique, écoulements.

Key-words : Particle size distribution, Taphonomy, Palaeolithic, Water flows.

\begin{abstract}
Abridged english version
PARTICLE SIZE DISTRIBUTION OF LITHIC ASSEMBLAGES AND TAPHONOMY OF PALAEOLITHIC SITES.

\section{1 - INTRODUCTION, METHOD}

The particle size distribution of lithic assemblages may provide valuable information on site formation processes. Once abandoned on the ground, the flint debris and used tools behave as sedimentary particles, that are susceptible to be redistributed by geomorphic processes untill their are completely buried. Superficial water flows are able to transport selectively the artefacts according to their size and/or shape, and as a consequence, to give birth to clear sortings. Recognition of such a sorting may have significant implications upon the interpretation of the artefact spatial distribution and, in some instances, of the techno-typological composition of the assemblage.
\end{abstract}

The method for grain-size analysis is derived from that used in sedimentology (sieving of the artefacts). To avoid damaging of fragil pieces, sieving can be replaced by manual measurement of their width with a ruler, taking in mind that a sieve mesh $d$ allows particles with a width I = $d 2$ to pass through due to their flat shape (fig. 1, 2, 4). Figure 3 gives the modified size class limits that have to be adopted for manual measurements. The use of the artefact number by size class rather than the weight is prefered here, since this allows giving emphasis on the fine-grained fraction of the assemblage and renders easier the determination of a deficit in this part of the distribution. To be representative, the grain-size distribution has to be established on a significant proportion of the total assemblage, recovered by sediment sieving (smaller sive mesh $=2 \mathrm{~mm}$ ).

(1) INRAP, Centre d'activité les Echoppes, 156 avenue Jean Jaurès, F-33600 Pessac

(2) Université Bordeaux 1, Institut de Préhistoire et de Géologie du Quatem a ire, UMR 5199 «PACEA», bâtiment de géologie, Avenue des Facultés, F-33405 Talence cedex

(3) Ministère de la Culture et de la Communication, Service Régional de l'Archéologie du Nord-Pas-de-Calais, Ferme

Saint-Sauveur, avenue du Bois, 59650 Villeneuve d'Ascq cedex 


\section{2 - RESULTS}

Experimental debitages (Stahle and Dunn 1982 ; Hansen and Madsen 1983 ; Schick 1986 ; Patterson 1990 ; Lenoble 2005, and this paper) show that the number of items decreases exponentially with increasing size, the distributions being very similar whatever the debitage type (table 1, fig. 6). Differences have been found according to raw material type, debitage type, size of the end products and the flint knapper skill (fig. 8, 9). However, these are minor, and do not exceed $15 \%$ for a given particle size class. Comparison between archaeological assemblages and experimental data indicates underrepresentation or conversely, overrepresentation of a particular size class, that may testify to size sorting. This could be anthropogenic or sedimentary in nature.

Experiments on size sorting due to water flows (overland flow, river) have been made amongst others by Schick (1986) and Lenoble (2005). The main characteristics of the resulting size distribution allow distinction between pavements (i.e. residual sites), proximal and distal accumulations of archaeological material (fig. 16, 17, 18). Natural size sorting mainly affects the finer-grained fraction $(<5 \mathrm{~mm})$ by opposition to anthropogenic ones. The latter are due to 1) importation and/or exportation of items like prepared cores and finished tools, 2) uneven spatial distribution of the activities of tool production and use within the occupation area. The analysis of a well-preserved epipalaeolithic site (Port-de-Penne), where different activities have taken place (butchery, core reduction, repairing of hunting arms...) (fig. 20), shows that the whole lithic assemblage retains a grain-size distribution close to that of experimental debitages, while the artefact sets coming from each square meters depart significantly from the experimental distribution, the more the number of artefacts is low (fig. 21).

Unambiguous determination of the origin of size sorting is not always easy. In order to minimize the uncertainties that factor into the interpretation, the grain-size analysis has to be compared with other lines of evidence such as geology, fabrics, and surface aspect of the artefacts. This allows usually robust assumptions on site formation processes to be made.

\section{3 - ARCHAEOLOGICAL CASE STUDIES}

Examples of particle size analyses of French palaeolithic assemblages demonstrate the potential of the method to understand site formation processes. Les Bosses (Jarry et al. 2001) is a Middle Palaeolithic site located near Cahors (fig. 19). The lithic material is included in a gravel pavement on a colluvial cone. Grain-size analysis indicates a significant impoverishement in fine- to mediumsize fraction (fig. 23), resulting from sorting due to overland flow. This is associated with evidence of edge damage of the artefacts, low reffitting rates ( $1.8 \%$ for flint and $4 \%$ for quartzite) that suggests artefact transport by flow away from the site, and lack of clear spatial organisation of the level probably due to strong artefact diffusion.

Hermies-Le Tio Marché is a Middle Palaeolithic site (Vallin and Masson 2005) located in a small dry valley cut in loessic deposits. Clear artefact concentrations have been identified, that correspond to knapping spots and accumulations of Levallois flakes (fig. 24). Grain-size analysis of samples coming from artefact concentrations lying on the bottom of the valley shows very similar distributions, caracterised by a strong excess in fine-grained elements $(2-4 \mathrm{~mm}$ ) (fig. 21). While trampling may be responsible by part of this excess, microscopic examination of the small fraction shows a wide range in the degree of alteration (fig. 26, 27, 28, 29, 30), that highly suggests a complex history of the level, and probably, secondary accumulation of fine flint particules washed from the upslope part of the site.

\section{4 - CONCLUSION}

The grain-size analysis of lithic assemblages is an efficient tool to understand the site formation processes. It is based on the comparison between the grain-size distribution of archaeological assemblages and that of experimental debitages. Significant difference between the distributions reflects size sorting. If the experimental data set is already large enough to evaluate the variability due to the debitage and raw material types and makes it possible to identify unambiguously a size sorting, determination of the origin of sorting, i.e. natural or anthropogenic, can be not easy. This results mainly from the small number of available data on the grain-size variability in an occupation level due to an uneven spatial distribution of the activities of lithic production and use. However, in the present state of knowledge, robust inferences on site formation processes can be made when size sortings are associated with other evidence of overland flow and river action (geology, artefact distribution, surface aspect of artefacts...).

\section{Acknowledgements}

This paper is part of the program entitled "Taphonomy of Middle Palaeolithic assemblages in northern France" (L. Vallin, director). We acknowledge all the persons that have made the experimental debitages: B. Aubry, L. Bourguignon, M. Brenet, A. Delagnes, P. Fouéré, J. Pelegrin and V. Mourre. The archaeologists in charge of the studied sites (C. Fourloubey, M. Jarry and M. Lenoir) and the reviewers (J. Pelegrin, J.P. Texier et J. Tixier) are also thanked.

\section{1 - INTRODUCTION}

Une fois abandonnés sur le sol par les hommes préhistoriques, les débris provenant de la taille du silex et les outils usagés peuvent être assimilés à de simples particules sédimentaires, susceptibles de subir l'action des processus géomorphologiques jusqu'à leur enfouissement com- plet dans le sol. L'idée d'utiliser les vestiges lithiques pour déteminer si ces processus ont joué un rôle important dans la constitution d'un niveau archéologique est relativement ancienne et a fait l'objet de nombreux développements (Schick 1986 ; Kluskens 1990 ; Lenoble 2005). Plusieurs mécanismes sédimentaires ont la capacité de transporter sélectivement les particules en fonction de leur 
taille et/ou de leur forme. Ce tri granulométrique ou morphométrique est une signature qui peut être aisément mise en évidence par l'analyse de la composition des sédiments. La reconnaissance d'un tel tri dans une série lithique a d'importantes implications sur la signification que l'on peut donner à sa distribution spatiale et aussi, dans certains cas, à sa composition techno-typologique. Dans le cadre de ce travail, on présente un bilan des connaissances sur la distribution granulométrique des assemblages lithiques issues d'expériences ou de l'étude de sites paléolithiques. On tente simultanément de dégager les lacunes de nos données et de proposer des perspectives en vue de constituer un outil fiable au service de l'analyse taphonomique des sites paléolithiques.

\section{2 - MÉTHODE DE L’ANALYSE GRANULOMÉTRIQUE}

L'analyse granulométrique d'un sédiment consiste à séparer ses différents constituants en classes dimensionnelles à l'aide de cribles ou de tamis pour la fraction supérieure à $2 \mathrm{~mm}$, qui seule nous intéressera ici. En sédimentologie, on utilise de manière standard des tamis dont la maille suit une progression géométrique de raison 1,25 , soit $2 \mathrm{~mm}$, $2,5 \mathrm{~mm}, 3,15 \mathrm{~mm}, 4 \mathrm{~mm}, 5 \mathrm{~mm}, 6,3 \mathrm{~mm}, 8 \mathrm{~mm}, 10 \mathrm{~mm}$, $12,5 \mathrm{~mm}, 16 \mathrm{~mm}, 20 \mathrm{~mm}$ etc. En pratique, cette série est souvent simplifiée ; les mailles retenues sont 2, 4, 5, 10, 20 $\mathrm{mm}$. Pour une particule ronde, la taille de la maille correspond précisément au diamètre maximal susceptible de passer à travers. Pour une particule aplatie, cette taille ne comespond qu'approximativement à sa largeur. En effet, lorsque l'épaisseur est négligeable, la largeur maximale pouvant passer à travers une maille de taille $d$ est égale d 2 (fig. 1), soit $1,414 \mathrm{~cm}$ si la maille est égale à $1 \mathrm{~cm}$. Cette approximation est toujours négligée pour des sédiments composés de particules de forme généralement quelconque.

La nécessité de ne pas abîmer les objets archéologiques étudiés impose une procédure d'analyse différente. Si le recours au tamisage classique ne peut être évité pour la fraction la plus fine, les objets plus volumineux sont passés manuellement à travers les tamis, qui servent alors de gabarits, ou bien leur largeur est mesurée à l'aide d'une règle ou d'un pied à coulisse. La largeur correspond à la plus grande dimension de l'objet prise dans le plan ortho- gonal à son axe d'allongement et peut différer sensiblement de la " largeur" telle qu'elle est mesurée en archéologie, qui correspond à la plus grande dimension prise dans le plan orthogonal à son axe de débitage (Tixier et al. 1980). D'une manière pratique, la taille maximale des pièces que l'on soumet au tamisage dépend du temps que l'on peut consacrer à la mesure et des caractéristiques de l'industrie. Le nombre de petits objets étant souvent élevé, la mesure de la largeur des pièces inférieures à $1 \mathrm{~cm}$ devient rapidement très fastidieuse. Dans le cas d'industries lamellaires où certaines pièces de petite dimension ont un intérêt typologique ou technologique et peuvent être détériorées lors d'un passage au tamis, on préfère usuellement effectuer un premier tri de manière à sélectionner dans la fraction inférieure à $1 \mathrm{~cm}$ les objets remarquables pour les mesurer ensuite séparément, tandis que le reste du matériel est tamisé. Le raccord entre les données issues du tamisage et celles de la mesure manuelle de la largeur nécessite de déplacer les limites de classes granulométriques pour les secondes. Comme on l'a vu plus haut, la largeur maximale des pièces pouvant passer à travers un tamis de maille $d$ est comprise entre $d$ et $d 2$ selon leur forme. Dans le cas de matériel archéologique, une majorité d'objets ont une forme très aplatie et l'approximation faite pour les sédiments ne peut plus être considérée a priori comme négligeable. Une estimation de la valeur moyenne que l'on doit adopter pour pouvoir raccorder sans trop de distorsion les données du tamisage avec celles de la mesure de la largeur a été effectuée en comparant ces deux types de données sur des objets archéologiques (Aurignacien de Caminade, Lenoble 2005) et les produits d'un débitage Levallois expérimental. Dans les deux cas, le résultat indique que, pour obtenir le même effectif d'objets dans chaque classe dimensionnelle avec les deux méthodes, la largeur équivalente $I_{\text {eq }}$ à une maille de tamis $d$ donnée est très proche de $d 2$ (fig. 2). Les limites des classes dimensionnelles à adopter pour les objets mesurés manuellement deviennent donc $2,8 \mathrm{~mm}$, $3,5 \mathrm{~mm}, 4,5 \mathrm{~mm}, 5,7 \mathrm{~mm}, 7,1 \mathrm{~mm}$ etc. (fig. 3). Le diagramme triangulaire de la figure 4 illustre la distorsion si l'on ne prend pas en compte ce facteur correctif. Dans les séries données comme exemple, l'effectif de la classe [20 $\mathrm{mm}-31,5 \mathrm{~mm}$ ] mesuré manuellement est réduit de 3 à $18 \%$ par rapport à l'effectif obtenu par tamisage, tandis que celui de la classe $>50 \mathrm{~mm}$ est augmenté de 2 à $6 \%$.
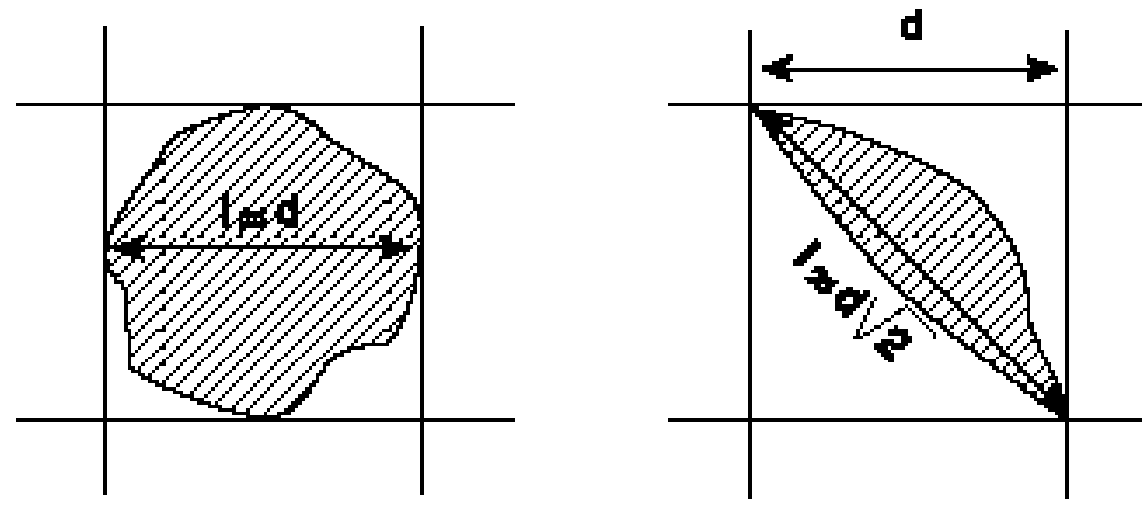

Figure 1 - Influence de la forme d'une particule sur la largeur maximale pouvant passer à travers un tamis de maille $d$.

Figure 1 - Influence of shape on maximum particle width able to pass through a sieve mesh $d$. 


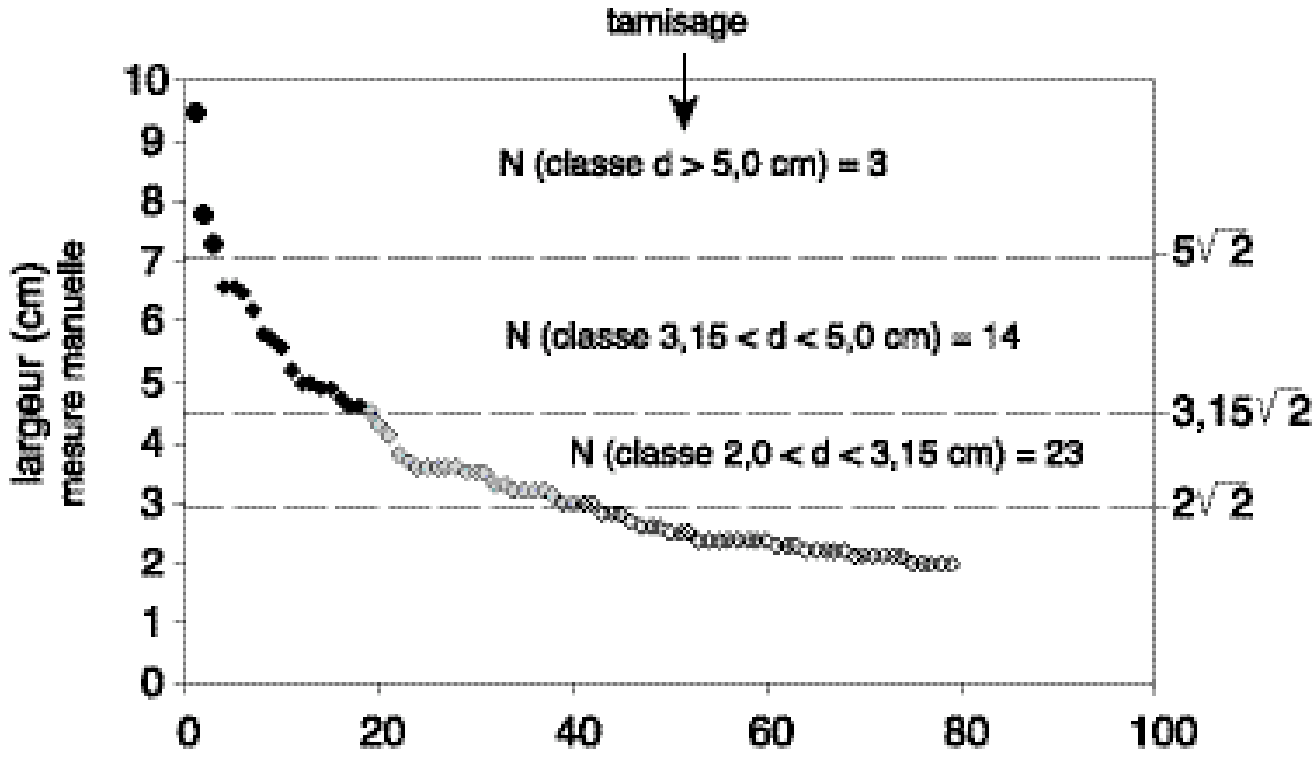

\section{Nombre d'objets}

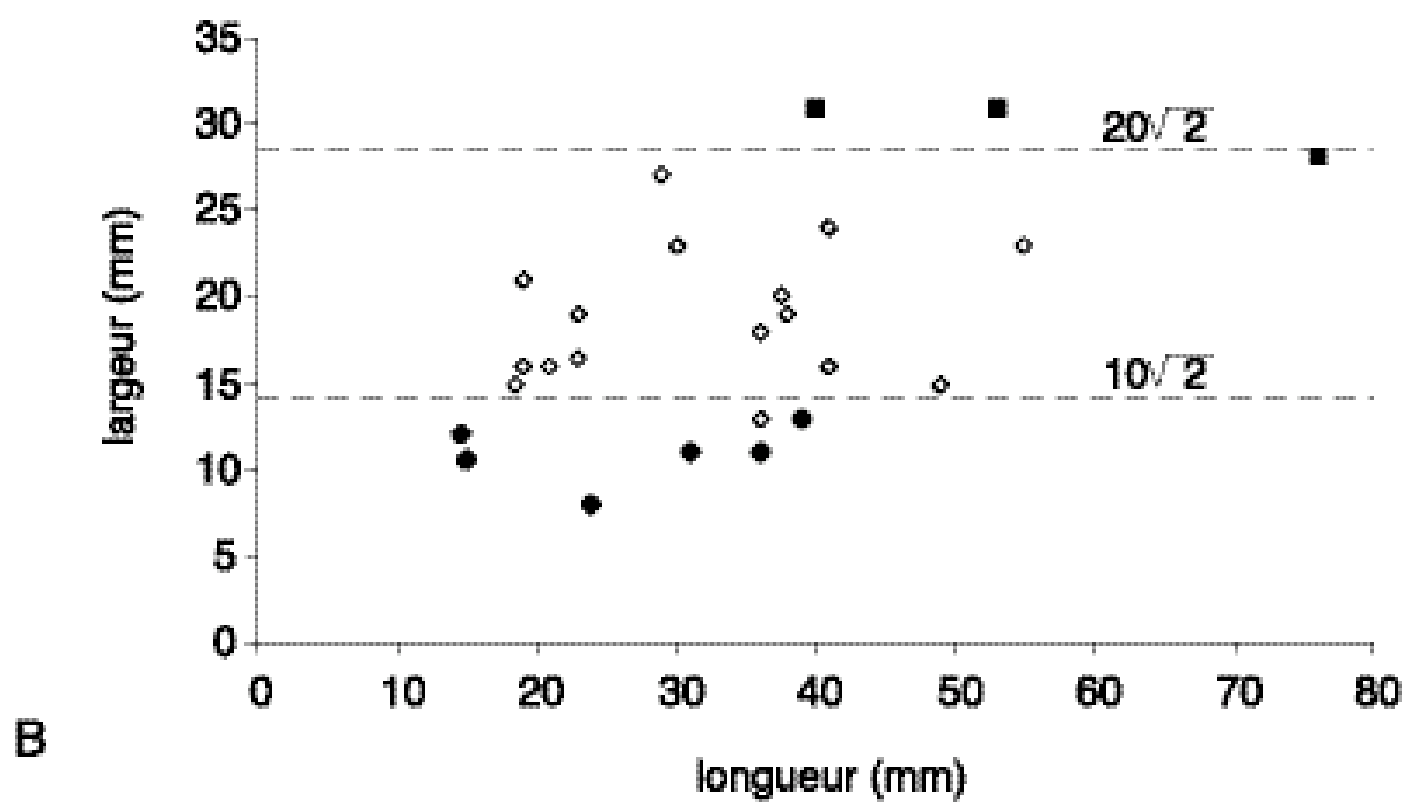

Figure 2 - A - Largeur (I) de 80 objets provenant d'un débitage levallois expérimental mesurés à l'aide d'une règle. L'appartenance des objets aux différentes classes de tamisage est indiquée par un symbole. Pour que les mesures correspondent au tamisage, les limites de classes doivent être fixée à I 2. B-Relation entre les dimensions d'une série d'objets aurignaciens (longueur, largeur) et les classes de tamisage, d'après Lenoble (2005).

Figure 2- $A$ - Width (I) of 80 artefacts from a levallois debitage measured with a ruler. The size classes are indicated by symbols. Similar results are obtained by measurement and sieving if the class limits are I 2. B-Relationships between length and width of Aurignacian artefacts and sieving classes, from Lenoble (2003). 


\begin{tabular}{|c|c|}
\hline$I(\mathrm{~mm})$ & $\sqrt{2}(\mathrm{~mm})$ \\
\hline 2 & 2,83 \\
2,5 & 3,54 \\
3,15 & 4,45 \\
4 & 5,66 \\
5 & 7,07 \\
6,3 & 8,91 \\
8 & 11,31 \\
10 & 14,14 \\
12,5 & 17,68 \\
16 & 22,63 \\
20 & 28,28 \\
31,5 & 44,55 \\
50 & 70,71 \\
\hline
\end{tabular}

Figure 3 - Valeur des limites de classes dimensionnelles à adopter pour comparer les largeurs mesurées et le tamisage.

Figure 3 - Size class limits that have to be taken to compare measurements and sieving.

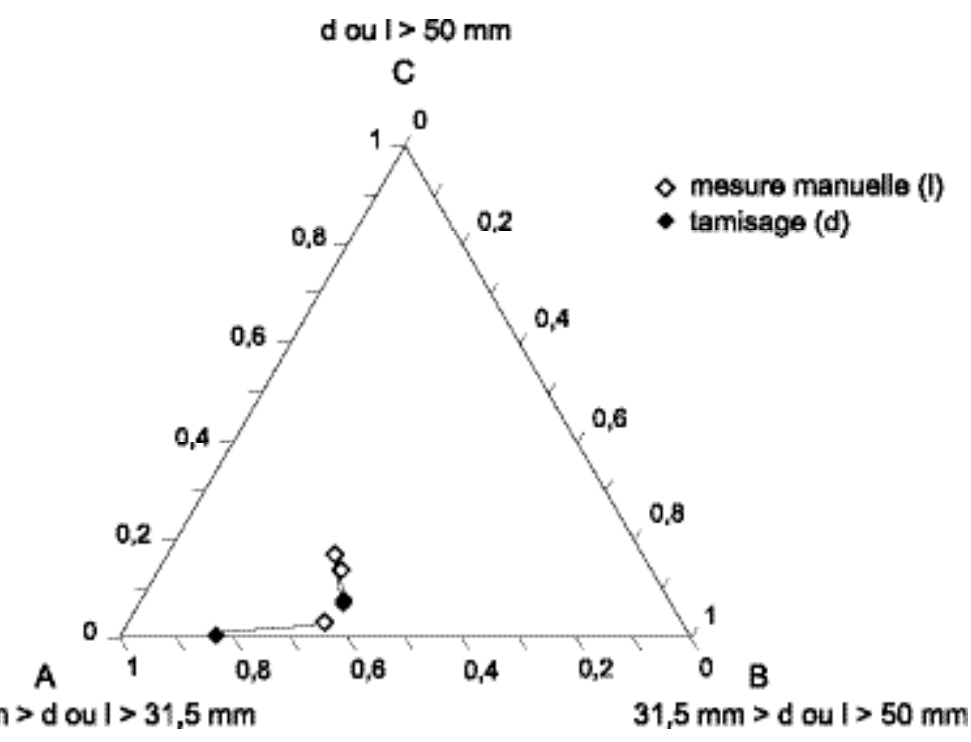

Figure 4 - Influence de la méthode de mesure de la largeur des objets (règle ou tamisage) sur la distribution granulométrique de trois séries lithiques paléolithiques.

Figure 4 - Influence of the granulometric method used (measurement vs. sieving) on the size distribution of three lithic assemblages.
Dans les paragraphes suivants, les limites de classes données correspondent aux mailles de tamis $d$.

Une fois le matériel réparti en différentes classes de taille, la distribution peut être quantifiée en mesurant le poids de chaque fraction ou le nombre d'éléments qu'elle contient. La première méthode est celle habituellement employée en sédimentologie. Dans le cas de matériel archéologique, la seconde méthode est préférable. L'allure d'une distribution pondérale est en effet très influencée par les éléments les plus volumineux qui sont également les moins nombreux. Dans le cas de séries peu importantes, de 50 à 200 pièces, une petite variation du nombre de grosses pièces se traduira par une modification importante de la distribution granulométrique. Cela a trois conséquences majeures :

1) Pour que la comparaison entre deux distributions pondérales soit valide, la quantité de matériel mesuré pour chacune d'elles doit être importante. Cela exclut donc les petites séries d'objets ou la pratique de tests sur des petites séries choisies au sein d'un niveau archéologique en fonction de la distribution spatiale ou de critères géomorphologiques. A l'opposé, une distribution numérale représentative pourra être établie sur de petites séries d'objets ( $N=100$ à 200).

2) Lors d'un transport par des écoulements, les éléments de petite dimension sont plus mobiles que la fraction grossière. La distribution granulométrique exprimée en poids est peu sensible à un appauvrissement modéré en petits éléments. A l'inverse, une distribution exprimée en nombre d'éléments fera apparaître très clairement de petites modifications dues à un tri sédimentaire.

3) Pour de nombreuses industries, la majorité des outils ou des supports d'outils est d'assez grande taille. Le nombre de ces objets dans une série lithique est tributaire des comportements humains et varie largement selon la fonction du site et le type de production et de gestion du matériel lithique par les préhistoriques. Par ailleurs, les dimensions de cette catégorie d'objets sont très contraintes par la qualité et le volume des blocs de matière première. A l'inverse, les petits éléments sont principalement des déchets de taille abandonnés à l'endroit où ils ont été produits. Dans le cadre de l'étude taphonomique d'un site, où le principal objectif est de déterminer l'influence des processus naturels dans la constitution du site, l'utilisation des distributions granulométriques numérales qui minimise la contribution de la fraction grossière, s'avère la plus adaptée.

Différents moyens de représenter et de caractériser une distribution granulométrique sont possibles. En sédimentologie, on utilise classiquement des histogrammes, des courbes cumulatives ou des diagrammes temaires pour représenter les distributions. A partir des courbes, on peut calculer graphiquement des valeurs caractéristiques, comme la médiane $\mathrm{Md}$ (ou $\mathrm{d}_{50}$ ), qui est la dimension pour laquelle $50 \%$ du matériel est plus fin, et des indices de classement qui expriment le caractère plus ou moins res- 

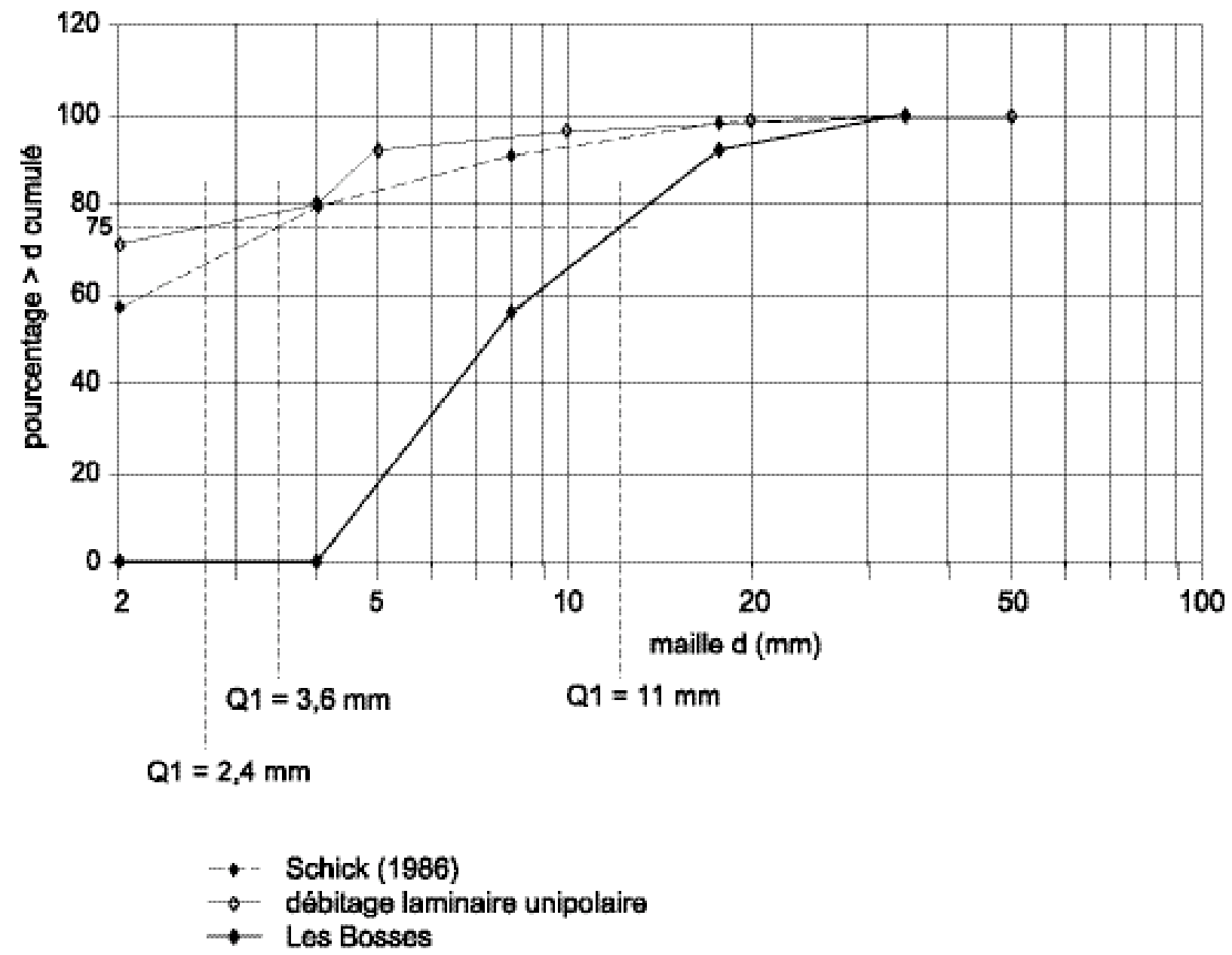

Figure 5 - Courbes granulométriques numérales cumulatives pour trois séries lithiques (débitage oldowayen, d'après Schick 1986, débitage laminaire unipolaire et série paléolithique moyen du site de Bosses, d'après Jarry et al. 2001). La valeur de Q1 est indiquée pour chaque série. L'échelle des abscisses est logarithmique.

Figure 5 - Cumulative particle size distribution (number of items by size class) for three lithic assemblages (oldowayan debitage, from Schick, 1986, unipolar laminar debitage, and Middle Palaeolithic assemblage of the 'les Bosses' site, from Jarry et al. 2001). Q1 is indicated for each artefact set. Logarithmic scale for $d(\mathrm{~mm})$.

serré de la distribution autour de la médiane. Les mêmes types de représentations peuvent être employés pour le matériel archéologique. Comme indice caractéristique de la distribution, la médiane a ici peu d'intérêt. En effet, cette valeur se situe presque toujours entre 2 et $4 \mathrm{~mm}$, la classe [2 mm - $4 \mathrm{~mm}$ ] rassemblant en général 60 à $80 \%$ des objets. Le premier quartile Q1 $\left(\mathrm{d}_{75}\right)$, c'est-à-dire la dimension pour laquelle $75 \%$ du matériel est de taille inférieure, est en revanche plus adapté. La figure 5 donne les valeurs de Q1 pour deux débitages expérimentaux et une série lithique paléolithique moyen.

\section{3 - COMPOSITION GRANULOMÉTRIQUE DES ASSEM- BLAGES LITHIQUES : DONNÉES DISPONIBLES}

Le corpus des données granulométriques concernant les assemblages lithiques est relativement réduit et hétérogène. Le principal facteur d'hétérogénéité des données disponibles tient au fait que les limites des classes granulométriques retenues sont différentes et que les variables prises en compte par les auteurs peuvent être alternativement la longueur des objets ou leur largeur et sont exprimées en poids ou en nombre d'objets. Ces données concernent également soit des niveaux archéologiques, soit des débitages et façonnages expérimentaux. Dans le cadre de ce travail, de nouvelles données ont donc été acquises selon le protocole exposé plus haut ; elles concernent surtout la granulométrie d'amas de taille réalisés par différents expérimentateurs (B. Aubry, L. Bourguignon, M. Brenet, A. Delagnes, P. Fouéré, J. Pelegrin et V. Mourre). Les produits de la taille ont été récoltés dans leur intégralité (taille sur une bâche plastique) puis tamisés, la plus petite maille de tamis adoptée étant de $2 \mathrm{~mm}$. 
Ces mesures ainsi que celles effectuées par Stahle et Dunn (1982), Hansen et Madsen (1983), Schick (1986), Patterson (1990) et Lenoble (2005) mettent toutes en évidence une distribution similaire des produits issus de la taille d'un bloc (tabl. 1, fig. 6). Elles montrent que la proportion de fragments décroît rapidement des petites vers les grandes dimensions, c'est-à-dire que le débitage ou le façonnage produisent énormément de petits débris et peu de grandes pièces. Cette distribution suit approximative ment une loi de type exponentielle décroissante (Schick 1986) ou plus exactement, selon Stahle et Dunn (1982), une loi de type Weibull qui se distingue d'une distribution exponentielle par son caractère asymptotique, le contenu des classes dimensionnelles les plus grossières tendant vers 0 . Très peu de différences ont été trouvées en fonction du mode de taille et des matières premières utilisées. Il est probable qu'il s'agit là d'une propriété inhérente à la fragmentation des roches dures. On peut par exemple remarquer sur la figure 7 la grande parenté entre les courbes obtenues par le débitage d'un bloc de silex et la fragmentation par le gel d'une paroi calcaire.

Bien que faibles, des différences ont néanmoins été observées (fig. 8, 9). En ce qui concerne l'influence de la matière première, notre corpus de données indique une tendance significative à une plus forte représentation de la fraction $2-4 \mathrm{~mm}$ pour le silex que pour le quartzite. Les moyennes sont respectivement de 67,9 \% $\pm 5,5(\mathrm{~N}=13)$ et $60,4 \% \pm 5,8(N=6)$. Schick (1986) observe également quelques différences entre les matières premières testées (quart, obsidienne, basalte, chert, ignimbrite). Les roches à grains grossiers (quartz, basalte), qui ont tendance à se fracturer de manière irrégulière, donneraient plus d'éléments de petite dimension que les roches à grains fins. En ce qui concerne l'influence du mode de débitage, quelques tendances nettes se dégagent également, bien

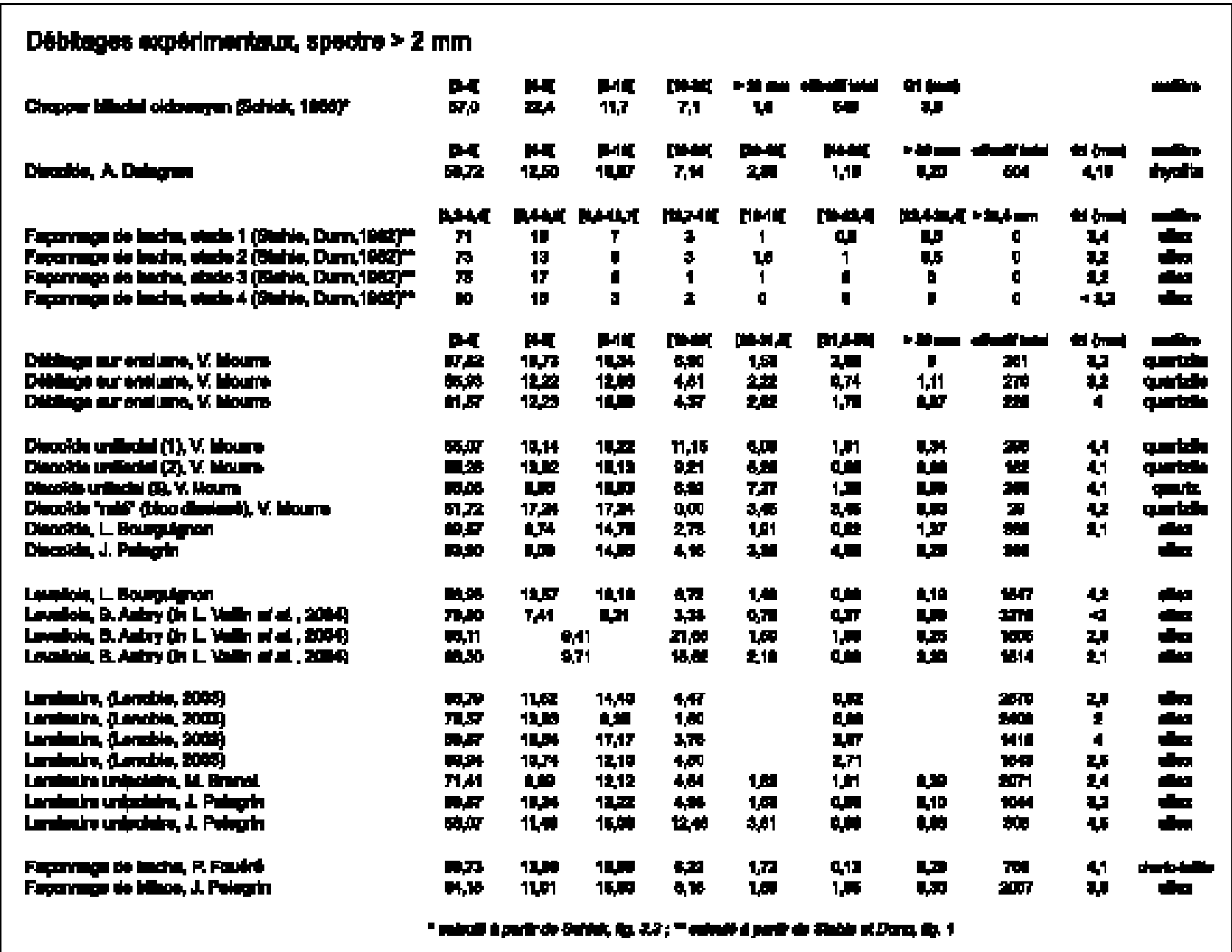



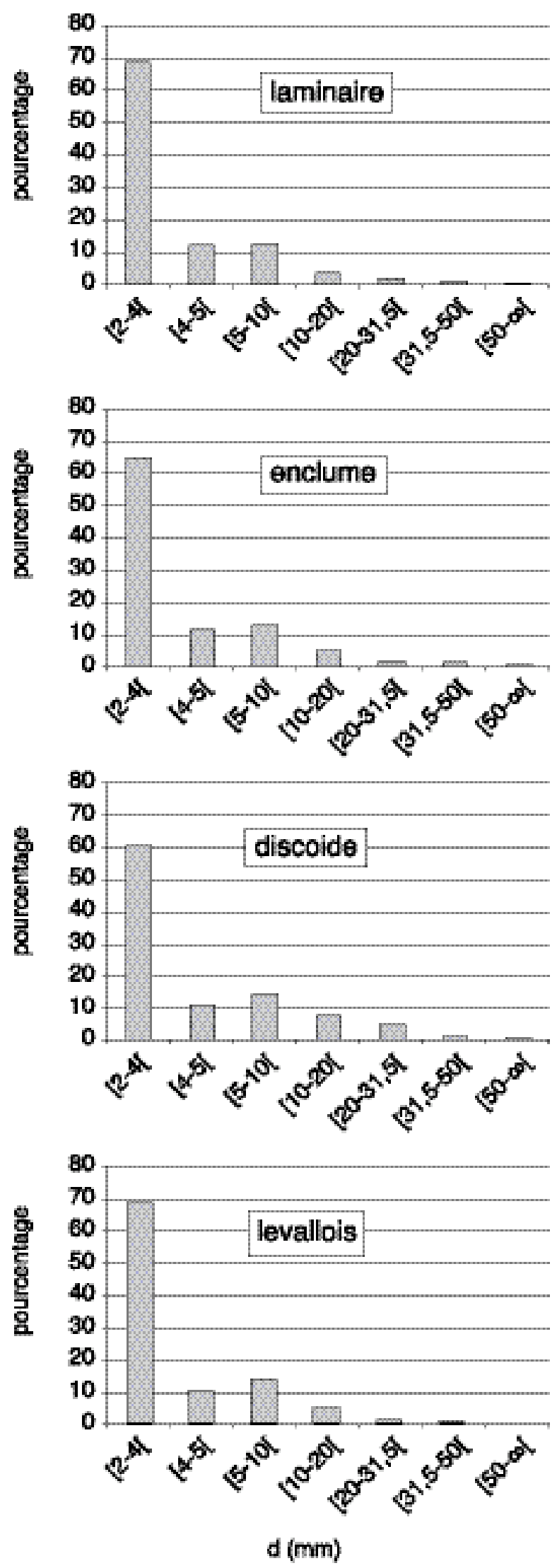

Figure 6 - Distribution granulométrique moyenne de quatre types de débitages expérimentaux. Noter que l'intervalle entre les limites de classes granulométriques n'est pas régulier (le "déficit" dans la classe 4-5 $\mathrm{mm}$ n'est donc qu'apparent).

Figure 6 - Mean particle size distribution for four experimental debitage types. Note that the intervals between the grain-size limits is not regular.

que notre échantillon expérimental soit relativement réduit. La proportion de la fraction $2-4 \mathrm{~mm}$ atteint environ $67 \%$ pour le laminaire $(\mathrm{N}=7), 65 \%$ pour le débitage sur enclume $(\mathrm{N}=3)$ et $60 \%$ pour le discoïde $(\mathrm{N}=6)$. Ces différences s'expliquent cependant en grande partie par le facteur matière première, puisque dans notre corpus, tous les débitages laminaires ont été réalisés sur silex alors que le quartzite domine largement pour les débitages discoïdes et sur enclume. Les deux exemples de débitage discoïde sur silex donne en effet une proportion de la fraction $2-4$ $\mathrm{mm}$ quasiment identique à la moyenne du laminaire, soit $67 \%$. Si l'on ne considère que les résultats obtenus pour le silex, les proportions des différentes fractions restent très voisines quel que soit le type de taille. D'autres facteurs, non explorés ici à défaut de données appropriées, sont également susceptibles d'influer sur la distribution granulométrique des produits :

1) la dimension des produits recherchés. Le façonnage de pièces bifaciales tend par exemple à ne produire que peu de grandes pièces (Patterson 1990), le but recherché n'étant pas l'obtention de supports d'outils. Les données de Stahle et Dunn (1982) indiquent un déficit en pièces de

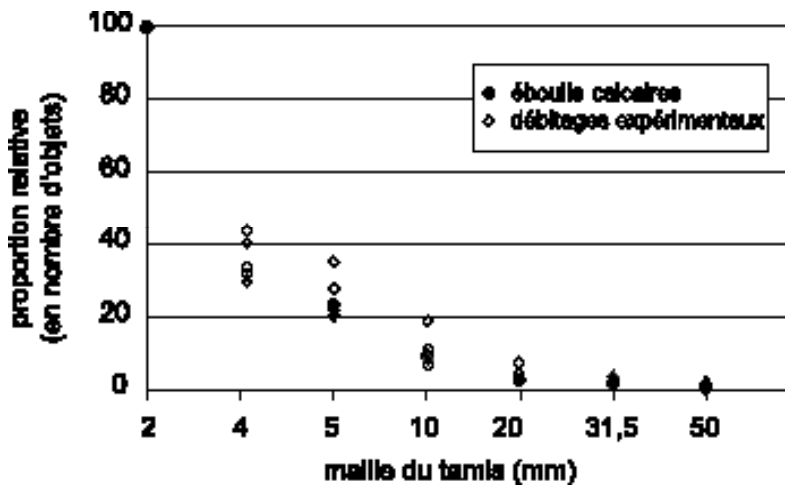

Figure 7 - Distribution granulométrique comparée de débitages expérimentaux et des produits de gélifraction d'une paroi calcaire.

Figure 7- Compared particle size distribution of experimental debitages and rock fragments derived from frost shattering of a limestone wall. 

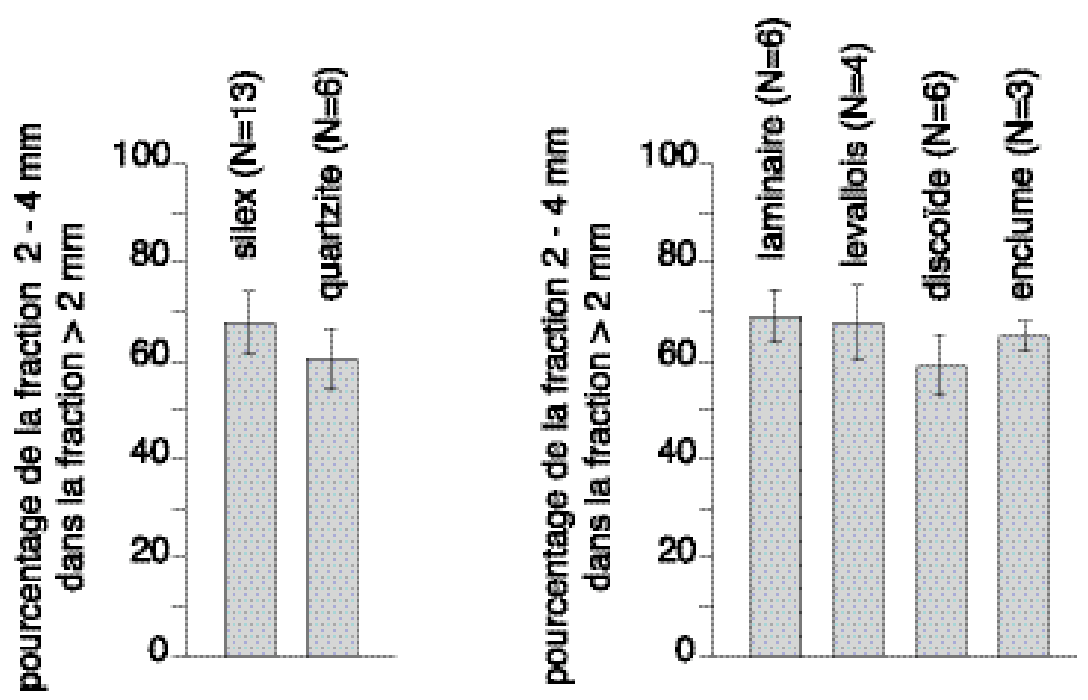

Figure 8 - Variabilité de la proportion de la fraction 2-4 $\mathrm{mm}$ en fonction de la matière première $(A)$ et du type de débitage (B).

Figure 8 - Proportion of the 2-4 $\mathrm{mm}$ fraction as a function of raw material $(A)$ and debitage types $(B)$.
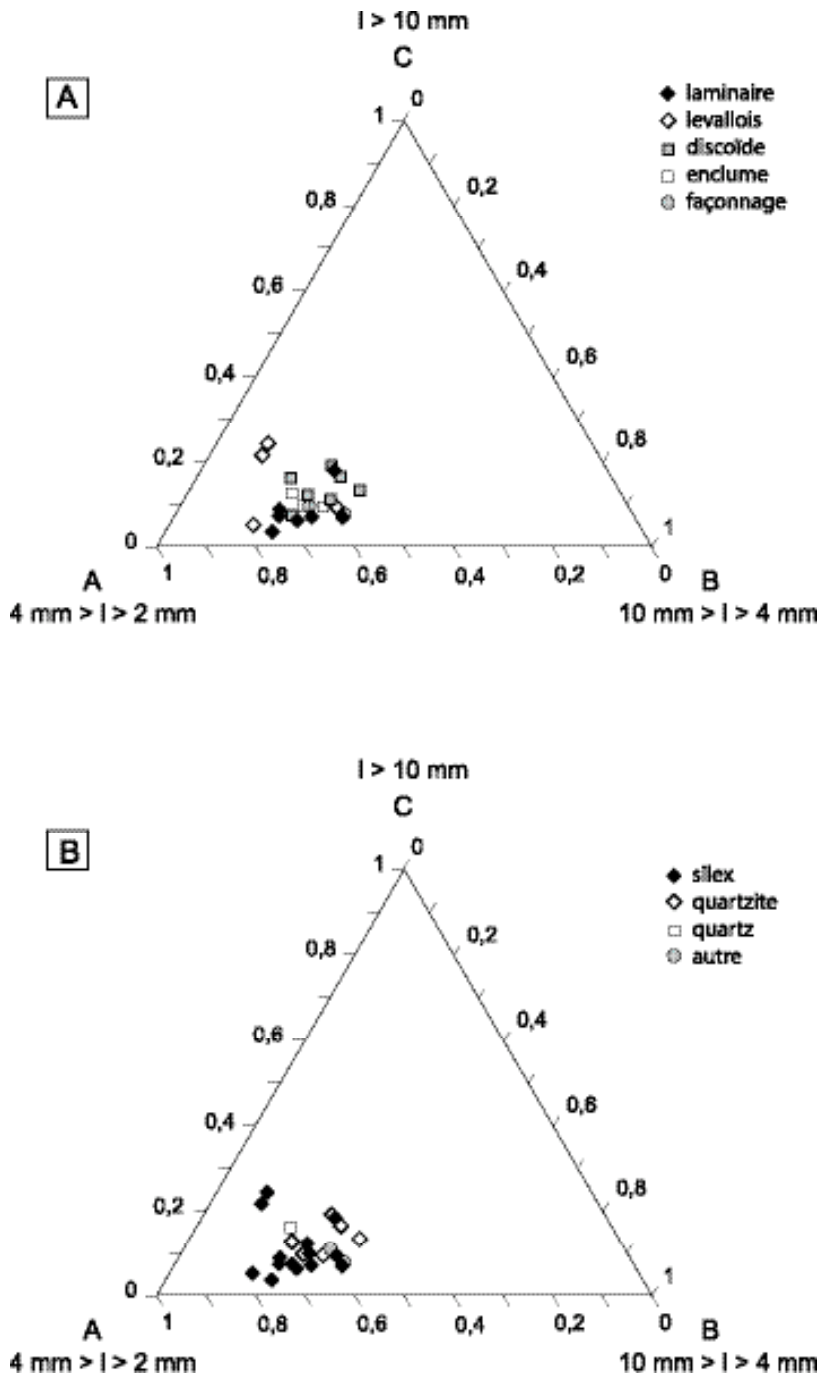

plus de $10 \mathrm{~mm}$ par comparaison avec le débitage discoïde d'autant plus marqué que la réduction de la préforme progresse. Les exemples disponibles dans notre corpus sont ceux du façonnage d'un biface en silex et d'une hache néolithique en cherto-tuffite. Les courbes obtenues rentrent cependant dans l'intervalle de variation des autres débitages. Par ailleurs, un débitage lamellaire à partir de petits nucléus ou d'éclats entraînera vraisemblablement une sous-représentation des classes grossières. Ce type de distribution n'est pas présent dans notre corpus ;

2) une préparation soignée des plans de frappe peut entrâ̂ner la production de petits éléments en quantité significativement plus élevée. Sur ce point, nos données indiquent que ce facteur n'a qu'un impact médiocre, bien que réel, sur la distribution granulométrique des produits obtenus. Ainsi, deux débitages Levallois réalisés avec une grande économie de gestes et une faible préparation des plans de frappe selon les modalités observées sur le site d'Hermies (Pas-deCalais) fournissent un pourcentage de la fraction $2-4 \mathrm{~mm}$ parmi les plus bas de l'ensemble de notre échantillon. Si les valeurs les plus élevées correspondent à des débitages laminaires pour lesquels la préparation des surfaces de débitage et des plans de frappe est poussée, les intervalles de variations des différents types de taille (débitages laminaire, Levallois, discoïde, façonnage, débitage sur enclume) se recouvrent cependant largement ;

Figure 9 - Composition granulométrique des débitages expérimentaux, diagrammes triangulaires. Les figurés correspondent aux types de débitages (A) ou aux types de matières premières $(B)$.

Figure 9 - Particle size distribution of the experimental debitages, triangular diagrams. Symbols refer to debitage types $(A)$ or to raw material types $(B)$. 
3) un autre facteur susceptible d'introduire une variabilité est la dimension des blocs de matière première. Ce facteur influencera nécessairement la taille maximale des pièces produites. Dans la mesure où les fractions les plus grossières ne comptent que peu dans l'allure de la courbe granulométrique numérale, on doit s'attendre à ce que ce facteur reste assez marginal lorsque l'on prend en considération l'ensemble de la fraction $>2 \mathrm{~mm}$;

4) pour un même débitage, la dimension des artefacts produits au cours des phases successives d'exploitation du nucléus peut varier sensiblement. Stahle et Dunn (1982) montrent ainsi qu'au fur et à mesure du façonnage de haches néolithiques, la proportion de la plus petite fraction $(0,32 \mathrm{~mm}-0,64 \mathrm{~mm})$ tend à s'accroître. La différence entre la première phase (décorticage du bloc) et la demière phase de façonnage atteint environ $8 \%$;

5) la maîtrise du tailleur peut également avoir une influence. Ceci est notamment suggéré par l'un des débitages laminaires réalisé par J. Pelegrin qui a fait preuve d'une grande économie dans la gestion du nucléus et a produit relativement peu de déchets tout en obtenant des supports laminaires bien calibrés. La proportion relative de la fraction 2-4 $\mathrm{mm}$ pour ce débitage compte parmi les plus faibles de tout notre corpus, soit environ $56 \%$. Cette proportion est $10 \%$ inférieure à celle obtenue pour les autres débitages laminaires ;

6) enfin, une fragmentation secondaire du matériel peut se produire sous l'influence du piétinement (Vallin et al. 2005). Ce phénomène peut prendre une certaine importance au niveau des concentrations de vestiges, où les objets sont en contact les uns avec les autres, mais il reste à quantifier précisément.

Dans l'état actuel de nos connaissances, la distribution granulométrique des produits de taille supérieurs à $2 \mathrm{~mm}$ apparaît donc relativement constante et sujette à des variations mineures, les différences de proportions pour chaque classe granulométrique étant toujours inférieures à $15 \%$ d'un débitage à l'autre. La courbe moyenne peut donc être considérée comme une bonne approximation de la distribution, quelque soit la matière première utilisée et le mode de débitage. Par conséquent, on peut considérer que tout assemblage lithique directement issu du débitage ou du façonnage d'un bloc et n'ayant pas subi de tri ultérieur, présente un type de distribution proche de cette courbe.

L'un des principaux écueils à l'utilisation de cette courbe granulométrique dans le cadre de l'étude d'un site archéologique est la nécessaire récolte exhaustive du matériel lithique. Cette étape implique un tamisage à l'eau soigné du niveau archéologique. Certains processus naturels, comme la bioturbation, ayant tendance à entraîner une dispersion verticale d'autant plus importante que les objets sont petits (Van Nest 2002), le tamisage doit concerner l'ensemble du volume contenant le matériel archéologique et non seulement le niveau matérialisé par les gros objets. Cependant, même si le tamisage est effectué dans les meilleures conditions, l'intégralité du matériel n'est probablement jamais récoltée. Une estimation du taux de récupération a été faite par $A$. Lenoble (2005) à l'occasion d'expériences en milieu naturel qui consistaient à disperser sur le sol un nombre connu de vestiges sur des surfaces d'une dizaine de mètres carrés puis à les récupérer après quelques mois d'évolution sous l'action du ruissellement. Dans le cas où aucun objet n'avait été transporté hors de la zone fouillée par les processus sédimentaires, le

Figure 10 - Distribution granulométrique de différents débitages expérimentaux, $A$ - fraction supérieure à $5 \mathrm{~mm}$, $B$ - fraction supérieure à $1 \mathrm{~cm}$. Certaines mesures ont été regroupées pour obtenir des effectifs suffisants.

Figure 10 - Particle size distribution of experimental debitages, $A$ - fraction $>5 \mathrm{~mm}, B-$ fraction $>1 \mathrm{~cm}$.

Debitages have been grouped to obtain large enough artefact sets.
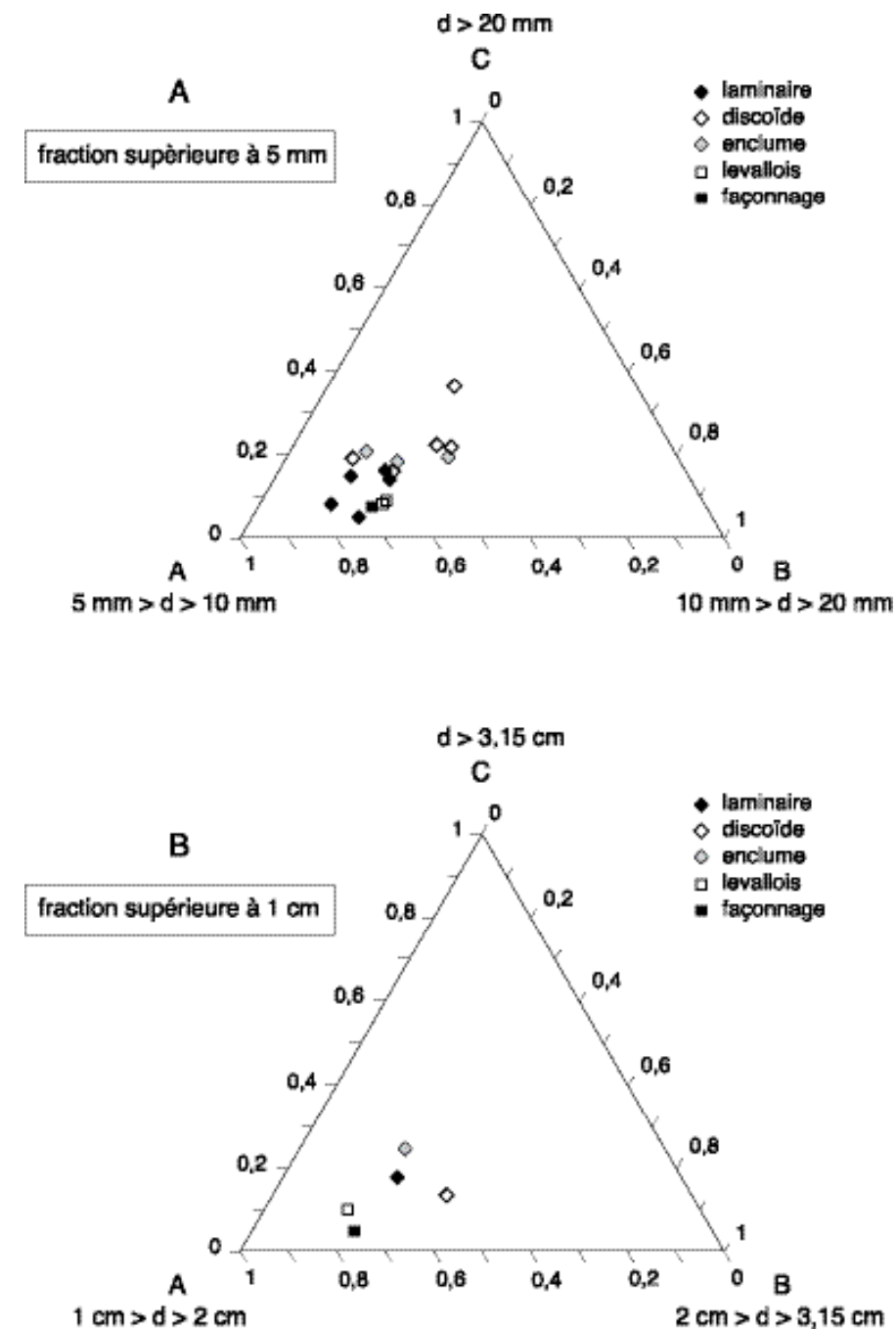


\section{Debilages experimentaux}

Spectre granulombtrique $>5 \mathrm{~mm}$

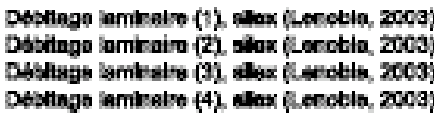

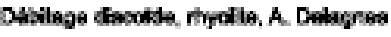

Dasituge su encume (1), quartibs, V. Moars Dobhtogo aur enolume t21. quartato, V. Mosne Dbsitngo sur anciume (3), quartzto, V. Mosico

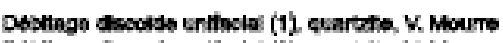
Dshitage drocoldo unitacial (2), cuartze V. MourTe

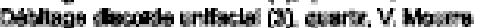

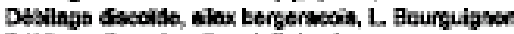
Dosttagn drocolda, slox, I. Priogt

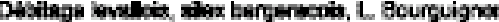

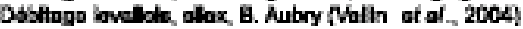

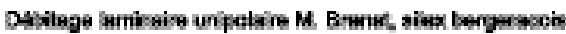

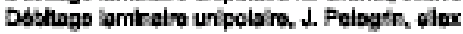
Dbstage iamiraive uripolaira, L. Pelogtr, alex

Facernaga oun biface, claxe, I Palecern

Faponnage ofuna bacto or othatoturito, P. Foubet

epoctro granulamátriquo > $1 \mathrm{~cm}$

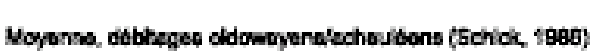

58,4

Dobthog lambato unipolairs, M. Bronos

Dsbithgo laminito unipotain, I. Palogtr

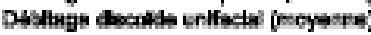

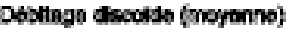

Dsbihngo Lovalois (moyerno)

Dbstags aur enciumb, $V$ Nourre (moyenne)

Faponnage on biface, L. Pwiwgrin

Faponnago do hacha, P. Foudré

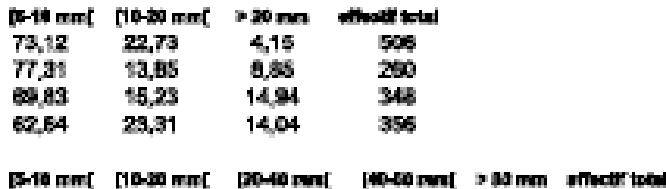

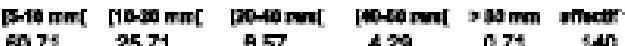

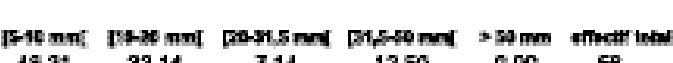

$\begin{array}{lllllll}48,21 & 32,14 & 7,14 & 12,50 & 0,00 & 50\end{array}$

$\begin{array}{llllll}50.32 & 22,03 & 10.17 & 3,30 & 5.09 & 50\end{array}$

$\begin{array}{llllll}60,33 & 18,87 & 10,00 & 8,57 & 3,33 & 00\end{array}$

$\begin{array}{llllll}48,60 & 32,04 & 17,48 & 2,91 & 0,97 & 103 \\ 48,64 & 29,79 & 17,02 & 2,43 & 2,13 & 47\end{array}$

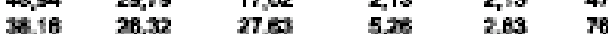

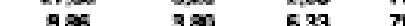

$\begin{array}{lllllr}66,35 & 12,56 & 8,85 & 3,90 & 5,33 & 70 \\ 88,86 & 16,39 & 12,50 & 17,31 & 0,96 & 104\end{array}$

$\begin{array}{llllll}66,12 & 26,47 & 5,41 & 320 & 0,71 & 425\end{array}$

$\begin{array}{llllll}\text { Co, } 81 & 24,12 & 5,75 & 2,05 & 0,80 & 452\end{array}$

$\begin{array}{llllll}60,82 & 22,02 & 9,22 & 5,10 & 1,24 & 412\end{array}$

$\begin{array}{llllll}63,13 & 23,75 & 7,83 & 4,15 & 0,46 & 217\end{array}$

$\begin{array}{llllll}45,00 & 38,00 & 11,00 & 2,00 & 2,00 & 88\end{array}$

$\begin{array}{llllll}62.85 & 24,90 & 5,83 & 4,22 & 1,20 & 488\end{array}$

$\begin{array}{llllll}0,57 & 22,71 & 5,23 & 4,22 & 1,20 & 480 \\ & 2,20 & 0,40 & 0,97 & 207\end{array}$

taux de récupération, c'est-à-dire le nombre de pièces retrouvées rapporté au nombre de pièces initialement déposées, a atteint $96,5 \%$ toutes fractions confondues. Pour la fraction comprise entre 2 et $4 \mathrm{~mm}$, ce taux était de $95,2 \%$, tandis qu'il était de $100 \%$ pour la fraction 5 - 10 $\mathrm{mm}$. Une valeur conservative de $90 \%$ peut être adoptée comme taux de récupération pour les fractions inférieures à $5 \mathrm{~mm}$ sur un site lorsque le tamisage a été effectué avec le dessein d'une collecte complète du matériel archéologique. Ceci signifie que des déviations de la composition granulométrique inférieures ou égales à $10 \%$ pour des séries archéologiques par rapport aux courbes expérimentales devront être considérées comme non significatives. Les résultats obtenus dans le cadre de cette étude sur des sites soigneusement tamisés valident cette hypothèse et indiquent que le biais lié à une récupération incomplète du matériel de petite dimension reste mineur. En effet, plusieurs ont livré une composition granulométrique similaire à celle du matériel expérimental, voire même plus riche en petits éléments. Un problème subsiste néanmoins lorsque l'on s'adresse à des sites fouillés anciennement, où le tamisage n'a pas fait l'objet d'une attention particulière. A titre d'exemple, le cas du site magdalénien de Moulin Neuf (abri 2) (Lenoir 1983), localisé dans un abri sous roche en Aquitaine, suggère en effet que le taux de récupération est souvent plus médiocre. La distribution observée montre un déficit très marqué en petits éléments qui atteint $54 \%$ pour la fraction 2-4 mm par rapport à la moyenne des débitages laminaires expérimentaux et $26 \%$ pour la fraction 4-5 mm, en considérant que les fractions plus gros- 
sières sont proches de leur proportion initiale. Ce déficit implique qu'un tri du matériel lithique s'est produit. Dans le cas présent, on suppose qu'il est dû à une récupération imparfaite des fractions fines au cours de la fouille, dans la mesure où elles ne présentaient qu'un intérêt technologique mineur.

De manière à disposer d'un référentiel mieux adapté aux sites pour lesquels la fraction la plus fine n'a pas été récoltée en totalité et pour répondre à des besoins spécifiques (voir infra), les distributions granulométriques expérimentales ont été recalculées en ne prenant en compte que la fraction supérieure à $5 \mathrm{~mm}$ (tabl. 2, fig. 10a) ou supérieure à $1 \mathrm{~cm}$ (fig. 10b). Dans ce dernier cas, plusieurs débitages ont été regroupés de manière à obtenir des effectifs d'objets suffisamment élevés. Les courbes obtenues sont globalement similaires aux précédentes. Néanmoins, l'amplitude des variations entre les différents débitages augmente. Ainsi, la représentation moyenne de la fraction 5-10 $\mathrm{mm}$ est d'environ $69 \%$ pour le laminaire, qui tend à produire des objets longs mais de faible largeur, tandis qu'elle n'atteint que $53 \%$ pour le discoïde et $57 \%$ pour le débitage sur enclume. En raison de ces variations, la comparaison avec une série archéologique nécessite que l'on prenne en compte le type de débitage. Les caractéristiques moyennes des courbes selon ce critère sont données dans le tableau 2.

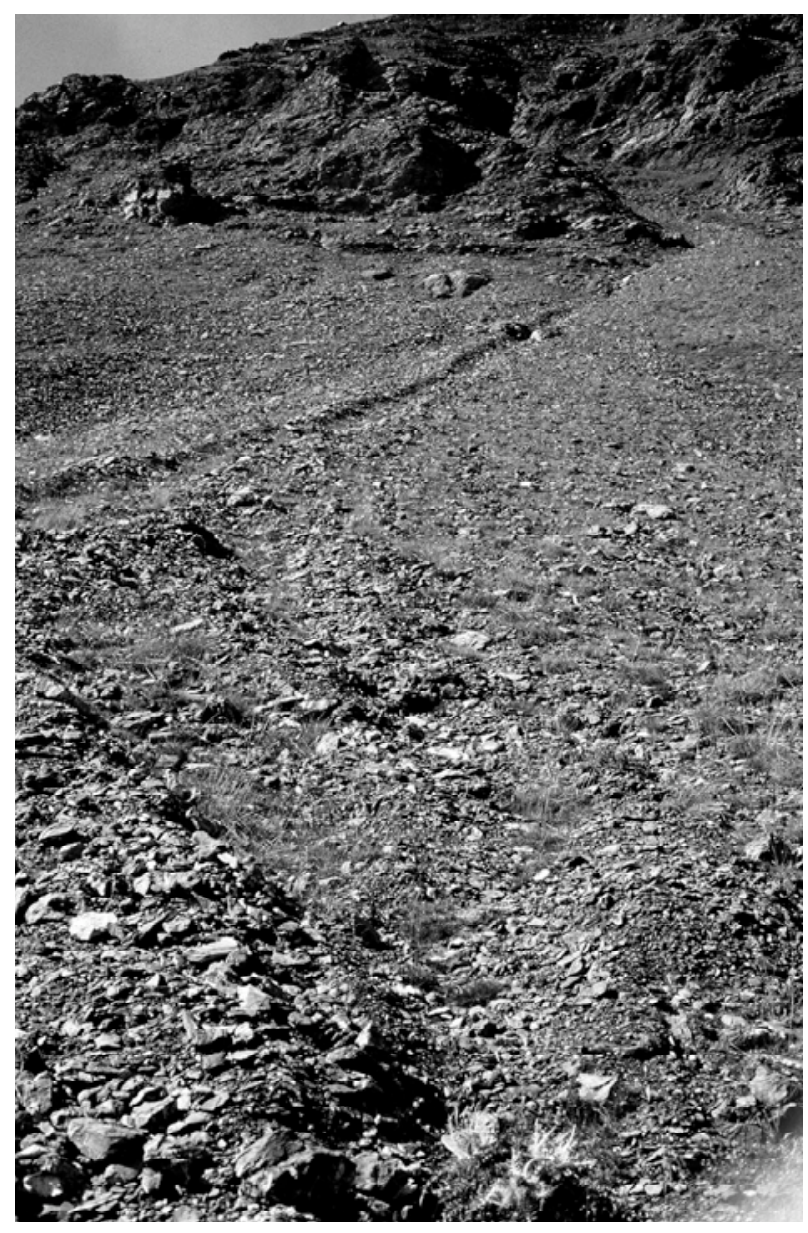

\section{4 - TRI GRANULOMÉTRIQUE : LE CORPUS DES DONNÉES GÉOMORPHOLOGIQUES ET LES EXPÉRIENCES ARCHÉOLOGIQUES}

Depuis Hjulström (1939), un nombre considérable de données expérimentales sur les relations entre les caractéristiques d'un écoulement et sa capacité à transporter des particules a été accumulé. En géomorphologie, on distingue habituellement plusieurs types d'écoulements :

1) les écoulements sédimentaires denses, qui contiennent une forte charge sédimentaire en suspension dans l'eau et ont une viscosité élevée,

2) les écoulements dilués, dans lesquels la charge transportée est peu importante par rapport au débit d'eau. Les premiers, qui se produisent typiquement sur des pentes fortes $\left(20-35^{\circ}\right)$, n'ont qu'une faible capacité de tri des particules (fig. 11). Les interactions électro-chimiques entre les particules argileuses et les frottements entre les éléments plus grossiers induisent un comportement " monophasique " de l'écoulement, c'est-à-dire que toutes les particules subissent un mouvement d'ensemble comme s'il s'agissait d'un matériel homogène. Le tri est donc très médiocre ; seuls les éléments les plus grossiers tendent à être expulsés en surface et à être poussés latéralement au fur et à mesure de l'avancée de la coulée. Très peu de données permettent de caractériser le tri qui en résulte. La figure 12 montre l'enrichissement relatif en petits éléments des levées d'une coulée de débris alpine de l'amont vers l'aval, soit sur une distance d'environ $150 \mathrm{~m}$.

Dans les écoulements dilués, les particules les plus fines (limons, argiles) sont maintenues en suspension par la turbulence, tandis que les éléments plus grossiers progressent sur le fond par roulement/glissement ou par saltation. Ces éléments sont alors respectivement en contact permanent ou intermittent avec le lit, l'ensemble de ces processus étant désigné sous le terme de charriage. La capacité d'un écoulement à entraîner du matériel dépend de la force tractrice qu'il exerce sur le fond. Cette force est une fonction de la vitesse du courant et de la rugosité du lit (Ramez 1995). Lorsque la granularité du lit est étendue, le contrôle de la mise en mouvement des particules est déterminé par les plus grosses, les grains plus petits étant bloqués par les premières. L'exposition des particules au courant joue également un rôle : la force exercée sur un grain dépassant d'un substrat plus fin est supérieure à celle qui s'exerce si le même grain est juxtaposé à des éléments de même dimension (phénomène de masquage). La capacité d'un écoulement à entrainer des objets est donc supérieure lorsqu'ils sont posés sur un fond sableux que sur un fond graveleux. Dans un cours d'eau, le transport et le dépôt des particules s'effectuent de manière intermittente, en fonction des variations du débit et des particularités topographiques locales du lit. Les formes de dépôt sont variables. Sur fond

Figure 11 - Coulée de débris (vallon Laugier, Alpes méridionales).

Figure 11 - Debris flow (Laugier valley, southern Alps). 

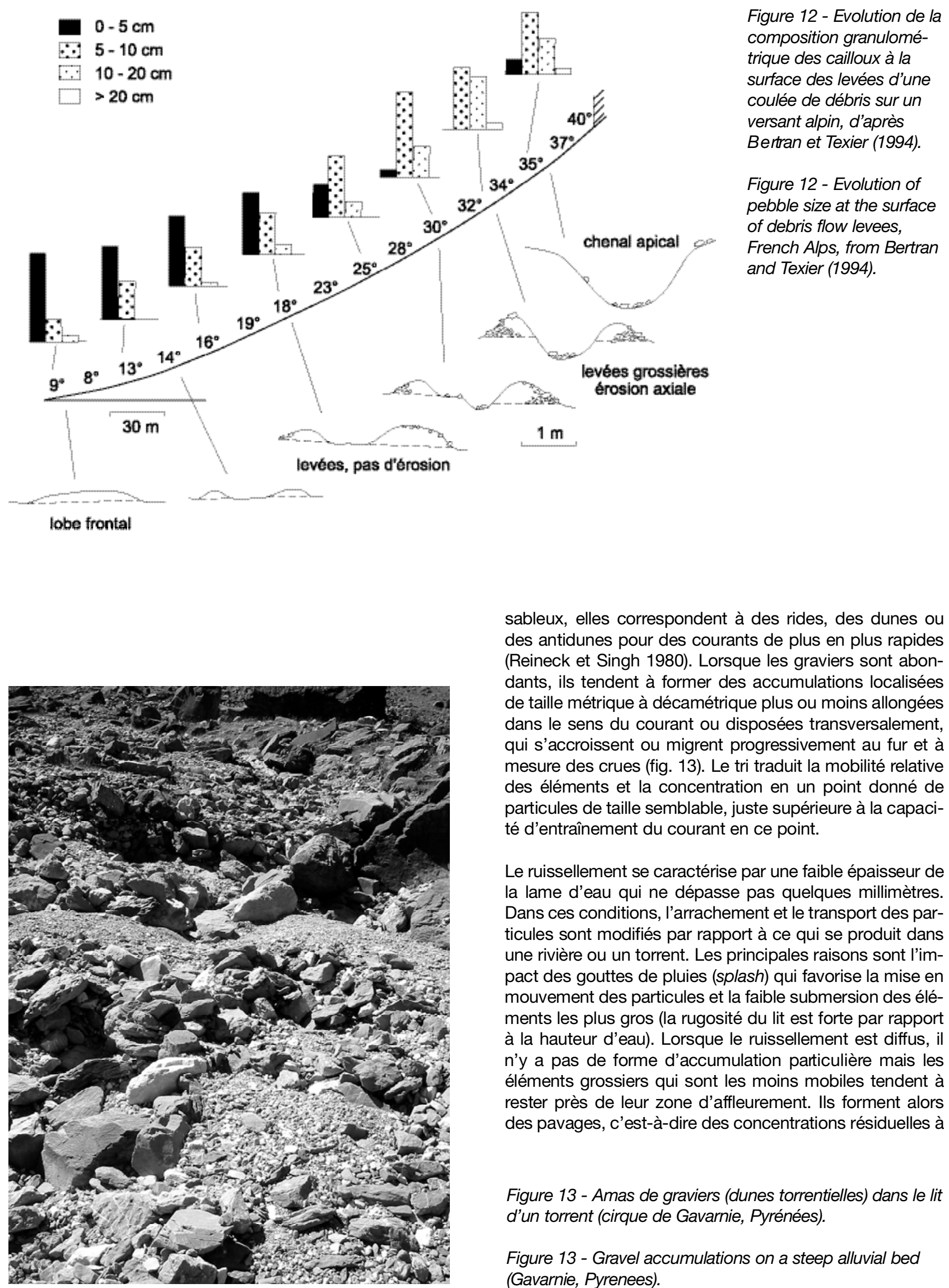

sableux, elles correspondent à des rides, des dunes ou des antidunes pour des courants de plus en plus rapides (Reineck et Singh 1980). Lorsque les graviers sont abondants, ils tendent à former des accumulations localisées de taille métrique à décamétrique plus ou moins allongées dans le sens du courant ou disposées transversalement, qui s'accroissent ou migrent progressivement au fur et à mesure des crues (fig. 13). Le tri traduit la mobilité relative des éléments et la concentration en un point donné de particules de taille semblable, juste supérieure à la capacité d'entraînement du courant en ce point.

Le ruissellement se caractérise par une faible épaisseur de la lame d'eau qui ne dépasse pas quelques millimètres. Dans ces conditions, l'arrachement et le transport des particules sont modifiés par rapport à ce qui se produit dans une rivière ou un torrent. Les principales raisons sont l'impact des gouttes de pluies (splash) qui favorise la mise en mouvement des particules et la faible submersion des éléments les plus gros (la rugosité du lit est forte par rapport à la hauteur d'eau). Lorsque le ruissellement est diffus, il n'y a pas de forme d'accumulation particulière mais les éléments grossiers qui sont les moins mobiles tendent à rester près de leur zone d'affleurement. Ils forment alors des pavages, c'est-à-dire des concentrations résiduelles à

Figure 13 - Amas de graviers (dunes torrentielles) dans le lit d'un torrent (cirque de Gavarnie, Pyrénées).

Figure 13 - Gravel accumulations on a steep alluvial bed (Gavarnie, Pyrenees). 


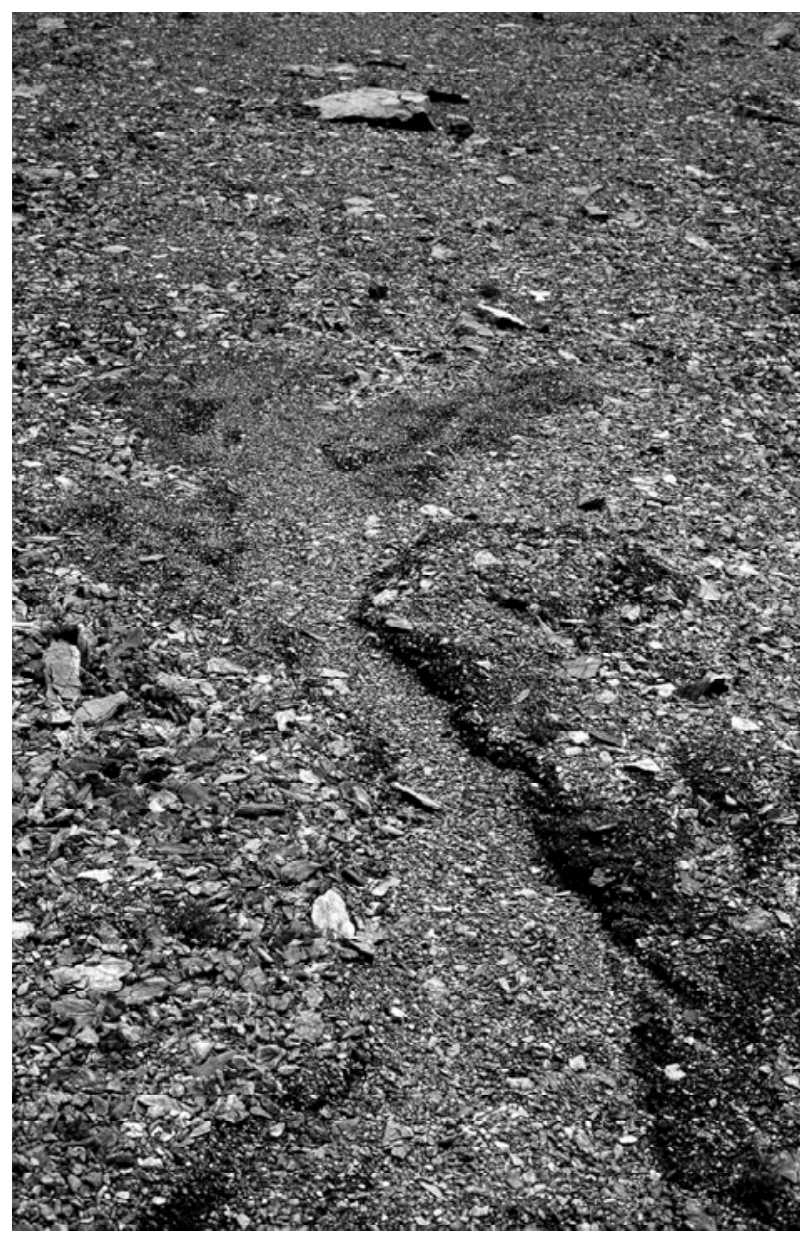

Figure 14 - Pavage de graviers et rigole (La Mortice, Alpes méridionales).

Figure 14 - Gravel pavement and rill (La Mortice, southern Alps). la surface du sol (fig. 14). Les éléments plus fins tendent à se concentrer vers l'aval et l'on observe un gradient granulométrique plus ou moins marqué d'amont en aval à l'échelle du versant dans les milieux où le ruissellement joue un rôle géomorphologique prédominant. Lorsque le ruissellement est concentré (rigoles), les formes d'accumulation s'apparentent à celles que l'on rencontre dans les toments. Le matériel grossier forme des accumulations localisées dans les rigoles, soit parce qu'il est trop volumineux pour être transporté et forme un pavage, soit parce que les éléments se sont bloqués les uns contre les autres pour donner naissance à des amas, alternant avec des épandages et des petits cônes sableux (fig. 15). L'échelle des formes d'accumulation est réduite et elles se succèdent rapidement à quelques décimètres de distance. Ces accumulations sont généralement moins bien triées que celles observées en contexte alluvial. L'un des principaux facteurs est le mélange de matériaux transportés sur une assez longue distance, qui ont donc subi un tri hydraulique, et de matériaux locaux qui n'ont pas été déplacés par les courants ou qui n'ont que brièvement été redistribués sur le versant.

L'une des difficultés, pour utiliser ces données en archéologie, vient du fait que ces distributions granulométriques comespondent la plupart du temps à des accumulations de matériaux qui ont enregistré un transport hydraulique pendant une longue période et qui ont subi plusieurs phases de remobilisation lors des crues avant d'être enfouis. Une occupation humaine se traduit en revanche par des apports de nouveaux matériaux dans un contexte sédimentaire où des tris peuvent déjà exister. Cette occupation ne sera reconnue comme site archéologique que dans la mesure où la dispersion des objets a été faible, ce qui implique dans la plupart des cas que le site n'ait pas été exposé aux écoulements pendant une longue durée mais ait été rapidement enfoui. Le recours à des expériences semble donc la meilleure approche pour caractéri-

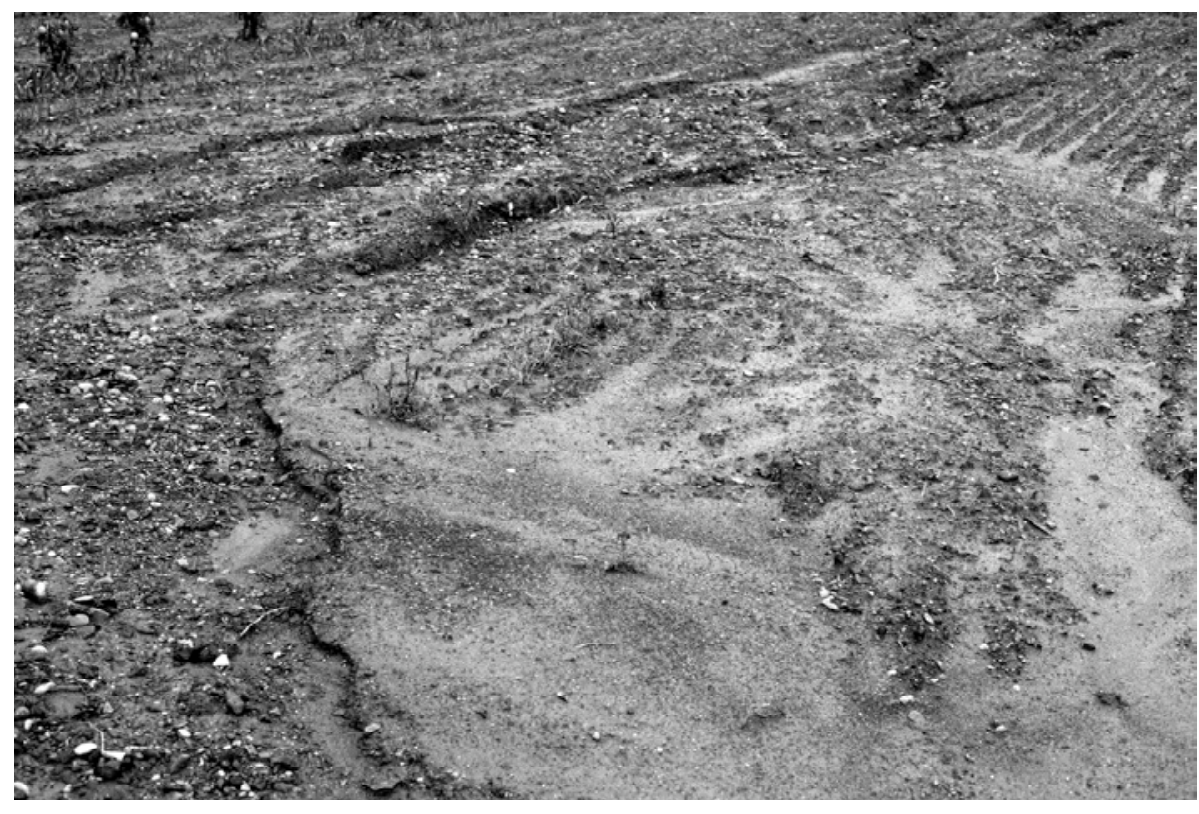

Figure 15 - Nappes de sables et de graviers au débouché d'une rigole (champ cultivé, région de Grenoble).

Figure 15 - Overland flow sand and gravel sheets (cultivated field, Grenoble region). 
ser les premiers stades de redistribution et obtenir des données utilisables en archéologie. Un autre point important est la morphologie généralement très aplatie des objets archéologiques (éclats, lames). Cette morphologie influe sensiblement sur leur comportement dans un écoulement, qui ne peut donc être directement comparé à celui des graviers naturels de forme arrondie. En l'état actuel, les données expérimentales concernant le matériel lithique sont assez réduites. L'essentiel provient des expériences de Schick (1986) en canal et dans des rivières en contexte semi-aride et celles de Frostick et Reid (1983), Petraglia et Nash (1987) et Lenoble (2005) pour le ruissellement.

L'influence de la morphologie des artefacts sur leur transport a été bien documentée par les expériences en canal réalisées par Schick (1986). Les objets épais, en particulier les nucléus, peuvent être aussi mobiles que des éclats de bien plus petite dimension parce qu'ils offrent plus de " prise " à l'écoulement. Par ailleurs, les objets anguleux ou de forme plano-convexe ont tendance à adopter une position stable, face plane vers le bas, qui les rend moins mobiles que les objets de forme approximativement sphérique (nucléus, choppers). Ces expériences, faites sur un fond sableux, indiquent également que l'interaction entre les objets joue un rôle important sur leur mouvement, soit parce que plusieurs d'entre eux se bloquent mutuellement, soit parce que les perturbations locales de l'écoulement autour des grosses pièces modifient les conditions de transportpour les plus petits objets. Tous ces facteurs permettent de penser que le tri granulométrique des objets archéologiques sera en moyenne plus médiocre que celui des matériaux alluviaux naturels où les galets sont de forme à peu près identique.
Les enseignements que l'on peut tirer de la vingtaine d'expériences faites par Schick (1986) en contexte alluvial naturel sont les suivants :

1) les modifications observées à l'occasion des crues sont très variables selon les sites, en fonction de leur localisation (plaine de débordement, berges, fond de chenal temporaire) et selon les caractéristiques des crues. Elles vont d'un enfouissement par des drapages de sédiment fin sans perturbation associée, jusqu'à des déplacements de grande ampleur (plusieurs dizaines de mètres) de la majorité du matériel. Le dépôt des objets transportés n'est pas aléatoire mais des zones de reconcentration locale sont observées.

2) la distance parcourue est principalement une fonction de la dimension des objets, le facteur " forme " ne jouant qu'un rôle secondaire. La granulométrie constitue donc le meilleur moyen de mettre en évidence un tri des objets.

3) Iorsque le matériel a été significativement remobilisé, les différentes zones de l'épandage final sont caractérisées par une composition granulométrique particulière. Dans le secteur amont de l'expérience baptisée " Flooded Workshop Site ", à l'emplacement du dépôt originel des objets, le matériel récolté est marqué par un fort appauvrissement en pièces de moins de $2 \mathrm{~cm}$ qui ne représentent plus qu'environ $20 \%$ du total contre $70 \%$ pour le débitage de référence. A l'inverse, nucléus et galets taillés comptent pour $50 \%$ du matériel récolté. Immédiatement à l'aval du site expérimental, la proportion d'objets inférieurs à $2 \mathrm{~cm}$ décroît encore jusqu'à une valeur quasiment nulle puis remonte progressivement en s'éloignant du site.

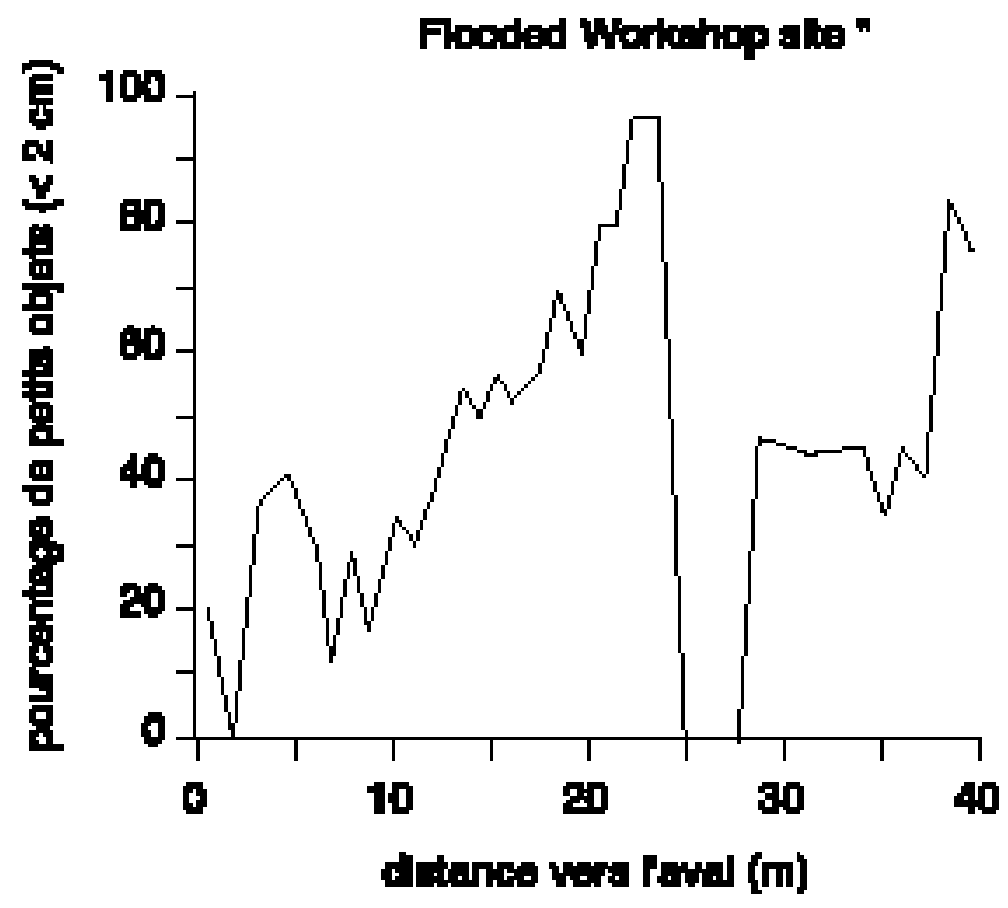

Figure 16 - Evolution de la proportion d'objets de moins de $2 \mathrm{~cm}$ dans un site expérimental redistribué en contexte fluviatile, d'après Schick (1986).

Figure 16 - Proportion of artefacts $<2 \mathrm{~cm}$ as a function of the distance from the original site on a river bed, from Schick (1986). 


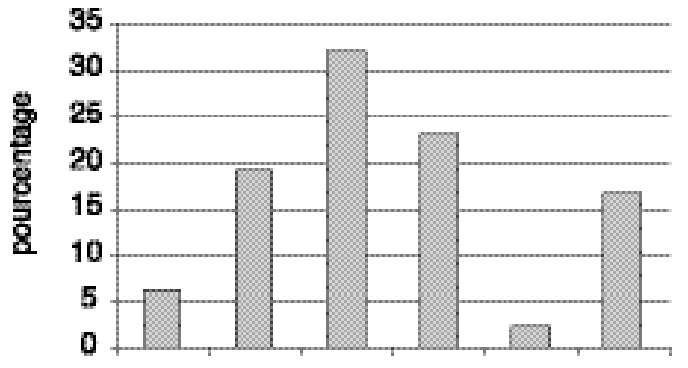

site résiduel
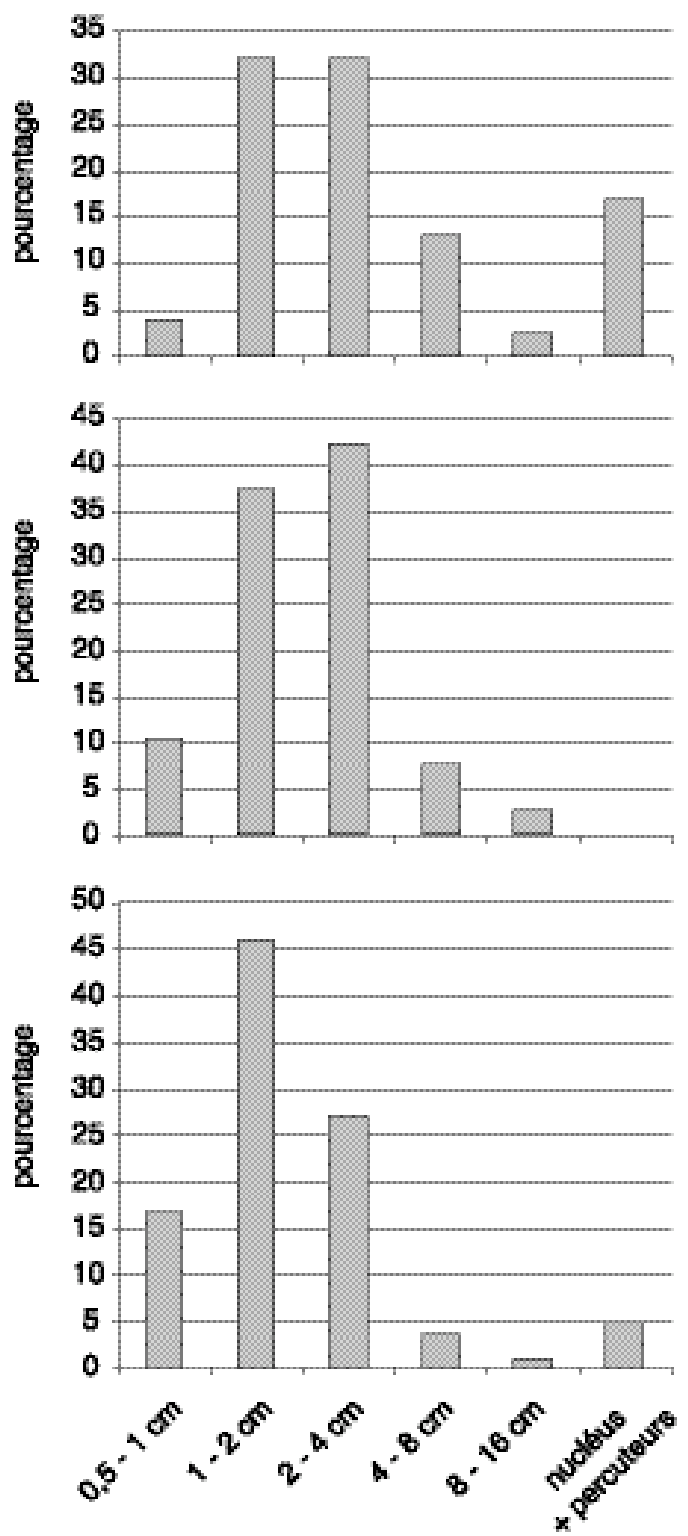

Figure 17 - Composition granulométrique d'une série lithique redistribuée par des crues, d'après Schick (1986). Les différents histogrammes présentent une allure en cloche caractéristique, dont le mode se déplace vers les petites dimensions au fur et à mesure que l'on s'éloigne de l'emplacement initial du site.

Figure 17- Particle size distribution of a lithic assemblage modified by floods, from Schick (1986). The histograms have typically a bell shape, the mode of which shifts towards small sizes as the distance to the original site increases.
Dans les secteurs plus distaux, c'està-dire à plus de $20 \mathrm{~m}$ du site, la fraction inférieure à $2 \mathrm{~cm}$ devient prédominante, pour représenter la quasi-totalité du matériel dans certaines zones de dépôt réparties de manière discontinue. Dans la partie la plus distale, les artefacts accumulés tendent à être sensiblement plus grossiers qu'un peu plus en amont. Cela a été attribué par Schick (1986) au fait que les petits objets, très minces, ont été enfouis plus précocement par les sables charriés sur le fond que les éléments plus épais. Les compositions qui caractérisent les différentes zones sont illustrées sur la figure 16.

L'ensemble de ces expériences montre que tout le spectre granulométrique d'une série archéologique peut être modifié très rapidement par les écoulements d'une rivière, même dans des contextes relativement peu énergiques (plaine de débordement faiblement végétalisée). Les redistributions affectent aussi bien les petits objets que le gros matériel et la signature de ces redistributions peut être mise en évidence à partir de ratios simples calculés sur le matériel supérieur à $1 \mathrm{~cm}$, tels que la proportion relative d'éléments inférieurs à $2 \mathrm{~cm}$ ou la proportion des éclats par rapport à celle des nucléus et autres gros objets (bifaces, choppers...). Les principales caractéristiques des distributions granulométriques sont (fig. 17) :

- à l'emplacement du site originel où l'assemblage est résidualisé, la distribution a une allure générale comparable à celle de départ dans les premiers stades de la redistribution (moins de 50 \% du matériel a été entraîné hors du site), c'est-à-dire de type exponentielle décroissante. La proportion relative des petits éléments est cependant significativement réduite. Pour des stades plus avancés de redistribution, la courbe tend à devenir plate, la proportion relative des fractions grossières dans l'assemblage final s'accroissant progressivement ;

- à l'aval du site, les objets déposés ont tous subi un transport hydraulique et la distribution a une allure " en cloche ", conséquence du tri. A proximité du site, la classe d'objets la plus représentée est située dans les grosses 


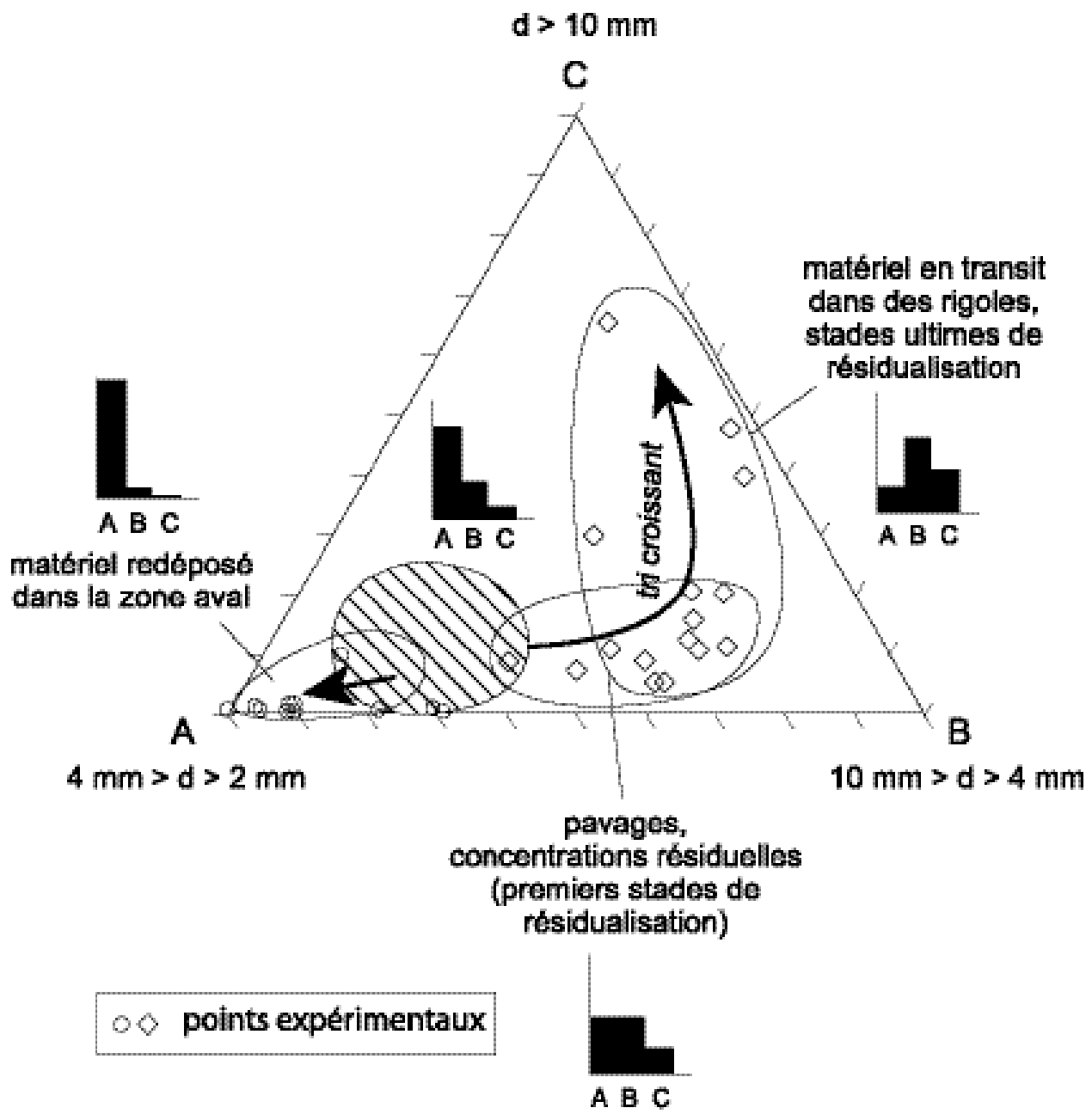

Figure 18 - Evolution granulométrique de séries lithiques soumises au ruissellement, d'après Lenoble (2005), modifié. L'aire hachurée correspond aux débitages expérimentaux.

Figure 18 - particle size evolution of lithic assemblages modified by overland flow, from Lenoble (2005). The hached area corresponds to experimental debitages.

A B C

dimensions (20-40 mm ou 40-80 mm). Le mode se déplace vers les petites dimensions lorsqu'on s'éloigne de la zone-source. Ces types de distribution évoluent peu lorsque l'intensité du transport s'accroît mais la quantité d'objets retrouvés par unité de surface diminue.

Pour le ruissellement, les séries les plus complètes d'expériences sont dues à Lenoble (2005). Les principales conclusions que l'on peut en tirer sont les suivantes :

- le ruissellement est capable de mobiliser tous les objets archéologiques, même les plus volumineux, qu'il s'agisse du ruissellement concentré dans des rigoles ou du ruissel lement diffus sur sol nu. Comme dans les expériences de Schick, la dimension des objets est le principal paramètre qui contrôle la distance parcourue, bien que leur forme joue un rôle non négligeable ;

- un tri granulométrique apparaît rapidement dès les premiers événements pluvieux. Ce tri augmente surtout en fonction de la durée d'exposition au ruissellement ;

- la variabilité locale des conditions d'écoulement étant très supérieure à celle qui existe en milieu fluviatile (variation rapide de la hauteur de la lame d'eau en fonction de la microtopographie, fluctuation rapide du ruissellement selon les variations d'intensité de la pluie et de l'état du sol), ce tri est plus médiocre. Pour une particule de dimension donnée, la distance parcourue au cours d'un événement est très variable. En conséquence, dans ces expériences, les éléments compris entre 2 et $4 \mathrm{~mm}$ sont toujours présents en quantité importante, non seulement dans les zones d'accumulation mais aussi dans les zones de départ et de transit des objets ;

- la disposition initiale des objets joue un rôle déterminant. Les amas tendent à dévier les filets d'eau et seuls les objets situés en périphérie sont mobilisés. La distribution granulométrique au sein de l'amas est donc peu modifiée dans les premiers stades d'exposition au ruissellement mais sa surface se réduit progressivement.

Les caractéristiques granulométriques des séries provenant des expériences de Lenoble (2005) sont illustrées sur la figure 18 . 


\section{5 - INTERPRÉTATION DES DISTRIBUTIONS GRANULOMÉTRIQUES DE MATÉRIEL LITHIQUE ARCHÉOLOGIQUE}

L'interprétation de la distribution granulométrique de séries lithiques archéologiques repose sur la comparaison avec le référentiel expérimental. La validité d'une telle comparaison nécessite bien évidemment que tout le matériel archéologique sur l'étendue du spectre granulométrique considéré ait été récolté, soit globalement, soit dans des zones test suffisamment riches pour disposer d'un effectif d'objets représentatif. Toute déviation de la distribution archéologique par rapport à la distribution expérimentale est alors susceptible de refléter un tri granulométrique. Selon l'étendue du spectre analysé, la distribution de référence sera la courbe moyenne de l'ensemble des débitages expérimentaux ou celle d'une catégorie particulière (laminaire, discoïde...). Le tri peut avoir plusieurs origines, soit anthropique et liée aux activités humaines qui ont présidé à la constitution de l'assemblage lithique abandonné sur le site, soit naturelle et provoquée par les processus sédimentaires qui ont affecté le site avant qu'il ne soit totalement enfoui.

Différents facteurs de tri d'origine anthropique peuvent intervenir et renvoient à la " fonction " ou au "faciès techno-économique " du site. Ils peuvent également être liés à la répartition spatiale différenciée des activités de production et d'utilisation des outils ou des supports d'outils à l'intérieur même du site. Certains sites, proches des sources de matière première, sont dominés par l'activité de production de nucléus, d'ébauches et/ou de supports d'outils. Les distributions granulométriques expérimentales donnent vraisemblablement une bonne idée du matériel présent sur ce type de sites. D'autres, éloignés des sources de matière, sont essentiellement des sites de " consommation ", avec peu de débitage ou de façonnage sur place mais avec de nombreux outils ravivés, raccourcis et/ou cassés. L'importation de nucléus, de préformes ou d'outils inachevés plutôt que de blocs de matière première brute va avoir pour principale conséquence une sous-représentation des produits de grande taille provenant du dégrossissage et des premières phases de façonnage ou d'exploitation du nucléus. L'importation d'outils achevés aura une conséquence inverse, c'est-àdire une surreprésentation de la fraction grossière. L'impact de ces facteurs peut être apprécié en croisant les données granulométriques avec les informations livrées par l'analyse technologique de la série lithique. Cependant, comme cela a été indiqué plus haut, ils ne devraient pas modifier significativement la distribution granulométrique numérale de la série lorsque l'on prend en compte l'ensemble de la fraction supérieure à $2 \mathrm{~mm}$. En effet, si l'on double le nombre d'artefacts de plus de $3 \mathrm{~cm}$ de manière à simuler le fait que la moitié des objets de grande taille ont été importés, on s'aperçoit que l'allure de la courbe granulométrique change peu car elle est surtout déterminée par les fractions les plus fines. En revanche, lorsque l'on ne prend en compte que la fraction supérieure à $1 \mathrm{~cm}$, l'influence de l'importation-exportation d'objets et de la forme sous laquelle la matière première a été intro- duite dans le site sera plus déterminante sur la distribution granulométrique du matériel. Dans ce cas, à l'exception des situations " extrêmes ", l'interprétation des déviations de la composition granulométrique par rapport à la courbe de référence nécessite alors de tenir compte de l'analyse technologique, sur le modèle décrit par Schick (1986).

L'autre source potentielle de tri granulométrique induit par les comportements humains est la présence dans un même site d'aires d'activité différenciées quant à la production et à l'utilisation du matériel lithique. A titre d'exemple, le spectre granulométrique livré par les amas de débitage sera sans doute différent de celui qui caractérise les aires où les outils ont été utilisés pour des taches spécifiques et éventuellement ravivés. Il a été également constaté dans des sites du Paléolithique supérieur que les postes de débitage, le plus souvent localisés autour de foyers, étaient périodiquement nettoyés, les déchets de taille étant alors rejetés en périphérie de l'habitat (Pigeot 2004). De nombreuses esquilles échappaient néanmoins à ces opérations de nettoyage et subsistaient à l'emplacement de leur zone de production. Bien que cela n'ait pas été formellement analysé, il est probable que la distribution granulométrique des amas de rejet soit déficitaire en petits éléments (en particulier dans le cas d'une évacuation manuelle des déchets l'un après l'autre, par opposition à la vidange de restes de taille accumulés dans une peau) et qu'à l'inverse, ces derniers soient surreprésentés autour des foyers. Tous ces aspects sont encore largement inexplorés.

Le site de Port-de-Penne (Lot-et-Garonne, fig. 19), un campement laborien installé en bordure du Lot et enfoui par des limons d'inondation (Detrain et al. 1996), a été analysé pour évaluer l'impact de ces facteurs potentiels de tri anthropique. Le contexte sédimentaire, très favorable à une bonne préservation des niveaux d'occupation, laisse à

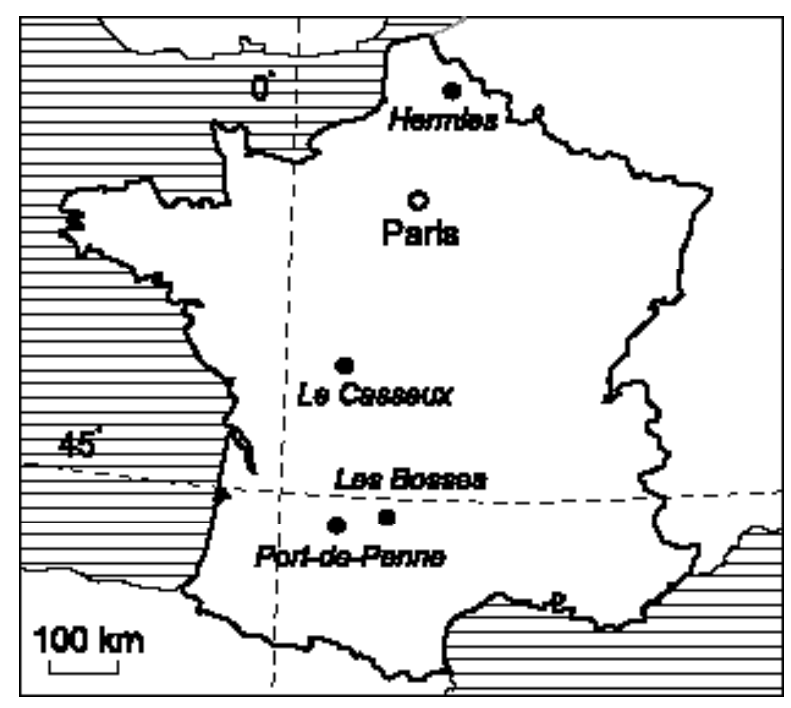

Figure 19 - Localisation des sites analysés.

Figure 19 - Location of the study sites. 

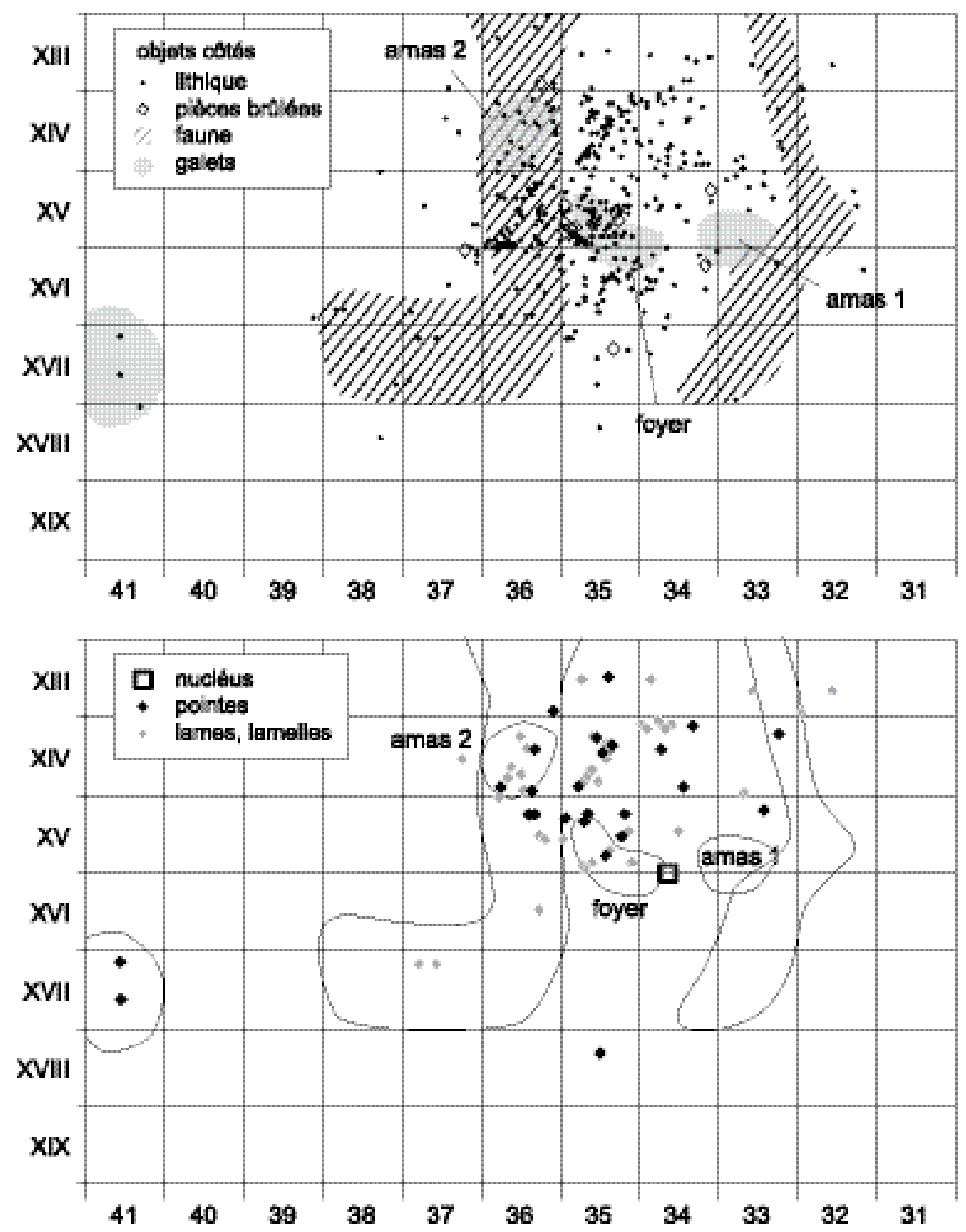

Figure 20 - Plans de répartition du matériel archéologique, site de Port-de-Penne, niveau 2, d'après Detrain et al. (1996). Sur la figure du bas, les lettres de a à $h$ renvoient à la position des échantillons granulométriques analysés et reportés sur la figure 21.

Figure 20 - Artefact distribution maps, Port-de-Penne, level 2 , from Detrain et al. (1996).

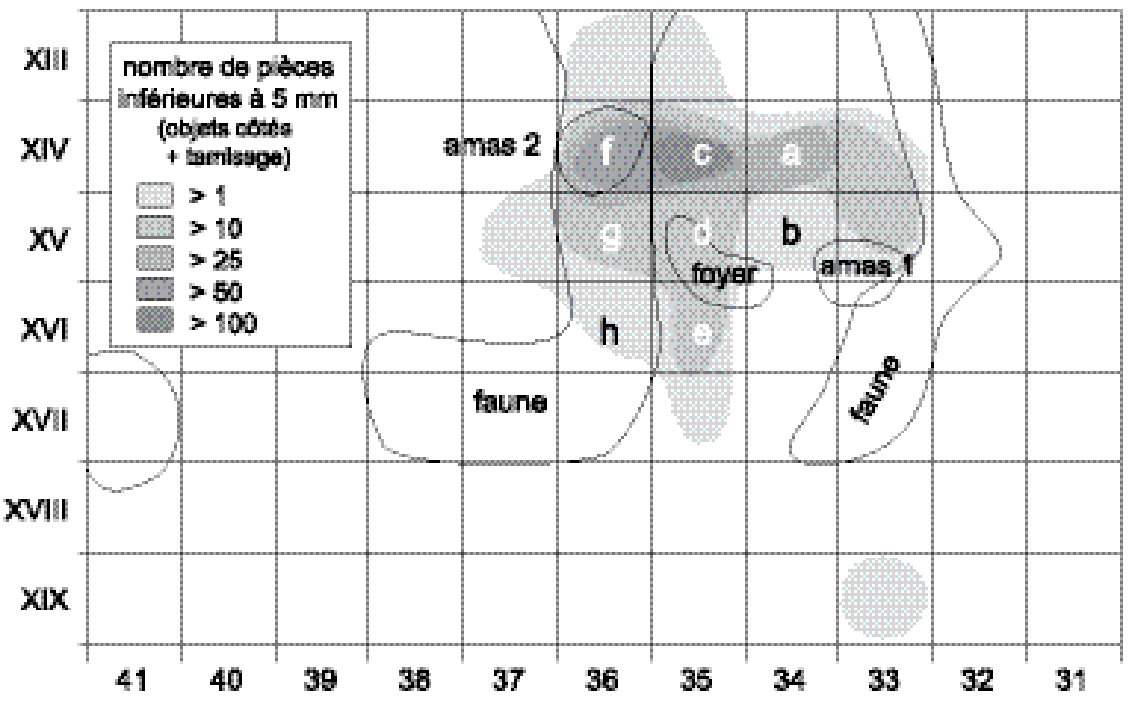

Port-de-Penne, niveau 2 


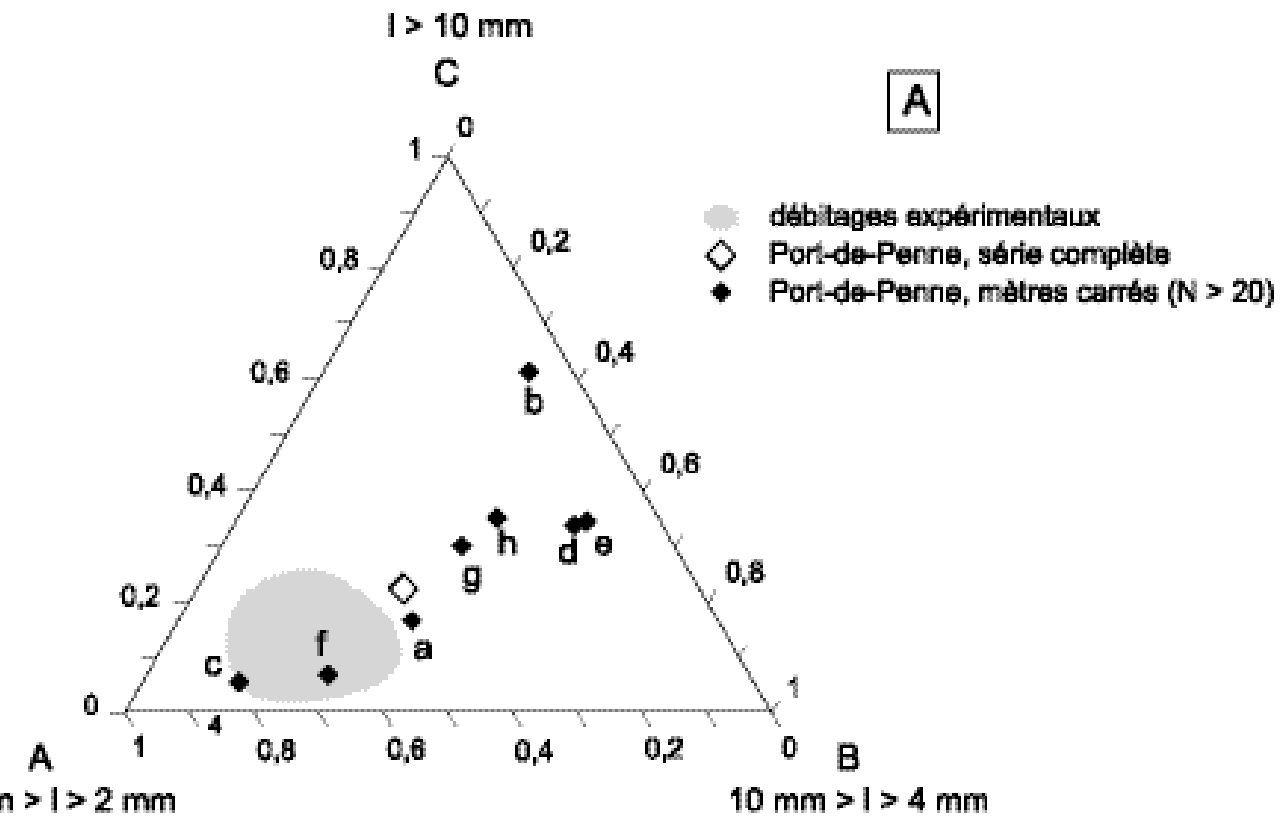

B

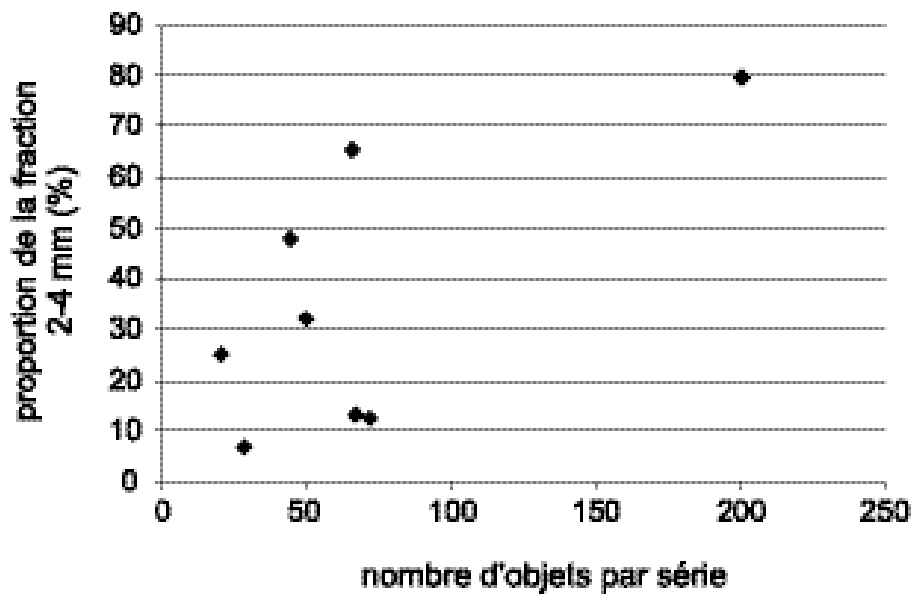

Figure 21 - Composition granulométrique de la série de Port-de-Penne, niveau 2.

Figure 21 - Particle size distribution of the lithic artefacts, Port-de-Penne, level 2.

penser a priori que les caractéristiques granulométriques du matériel lithique reflètent uniquement des phénomènes d'origine anthropique. L'analyse porte sur le niveau 2, dans lequel ont été mis en évidence un foyer, plusieurs amas de galets chauffés, 659 pièces de silex ainsi que de nombreux vestiges fauniques (cerf, cheval, bovinés et sanglier) concentrés dans deux cordons encadrant le foyer (fig. 20). L'étude technologique du matériel lithique indique que le débitage de supports laminaires n'a représenté qu'une activité secondaire et n'a pas donné lieu à la constitution d'un amas de taille, les différents produits étant dispersés sur une large zone comprise entre les cordons de restes osseux et le foyer. La matière première a été essentiellement introduite sous forme de nucléus déjà préparés et de produits finis, ce qui se marque par le caractère très incomplet des séquences de réduction. Le fort pourcenta- ge de grattoirs parmi les objets retouchés $(7 \%)$ et, surtout, celui des armatures de projectiles (pièces à dos : $62 \%$ et pointes de Malaurie : $20 \%$ ) suggère que ce campement était essentiellement tourné vers des activités de préparation du gibier et de réfection des armes de chasse. II s'agit donc d'un site spécialisé témoignant d'une occupation de courte durée, dans lequel seul un débitage d'appoint a été réalisé sur place. Malgré l'importation massive sur le site de supports déjà débités, la série lithique prise globalement possède une distribution granulométrique assez proche de celle des débitages expérimentaux (fig. 21A). Un déficit en petits éléments apparaît toutefois mais reste modeste (10 à $15 \%$ ). En revanche, lorsqu'on considère chaque mètre carré de manière isolée, des variations d'abondance très importantes de la fraction inférieure à 5 $\mathrm{mm}$ apparaissent. Une forte concentration de petits élé- 
ments (174 fragments $/ \mathrm{m}^{2}$ ) peut notamment être distinguée à proximité du foyer. Cette zone, qui comprend relativement peu d'objets de grande taille, a été interprétée comme une aire de confection et/ou de réaffûtage des pièces à dos, opérations qui ont produit une grande quantité de petits éclats de retouche. La comparaison entre les lots de plus de 20 objets récoltés sur $1 \mathrm{~m}^{2}$ montre que les écarts à la moyenne générale sont d'autant plus importants que l'effectif est faible (fig. 21B). L'étude de ce site suggère donc que :

1) les distributions granulométriques expérimentales sont utilisables comme base de comparaison pour un grand nombre de sites, même si le débitage et/ou le façonnage ne représente pas la totalité des activités pratiquées (sites intermédiaires entre sites de "production" et sites de " consommation "). Les séquences de réduction peuvent n'avoir été que très partiellement réalisées sur place ;

2) la présence d'aires d'activité bien différenciées à l'intérieur d'un site nécessite de multiplier le nombre d'échantillons, de manière à s'affranchir de la variabilité spatiale intra-site et à obtenir une distribution représentative.

De manière à limiter au maximum les incertitudes qui pèsent sur les caractéristiques originelles de la composition granulométrique des vestiges présents dans un site et qui affaiblissent sensiblement les possibilités d'interprétation des déviations par rapport aux distributions expérimentales, plusieurs recommandations peuvent être faites. Elles concernent d'une part, la méthode générale d'approche taphonomique d'un site, dans laquelle doit s'insérer l'étude granulométrique, et d'autre part, le choix des critères les plus solides, pour lesquels la variabilité d'origine anthropique est la plus faible.

La démarche privilégiée d'étude taphonomique d'un site est la " confrontation au modèle géoarchéologique ", telle qu'elle a été proposée par Collcutt et al. (1990) et Lenoble (2005). Elle procède par étapes, qui consistent ;

1) à identifier sur les coupes les mécanismes sédimentaires à l'origine du dépôt à partir des critères habituellement utilisés en géomorphologie, de manière à définir quels processus ont été susceptibles d'affecter les vestiges et de sélectionner les critères pertinents pour reconnaître leur action ;

2) à analyser les différentes caractéristiques des assemblages archéologiques récoltés dans ces dépôts (répartition spatiale, granulométrie, fabrique...) et à les comparer avec celles que l'on attendrait si l'organisation des vestiges était uniquement due aux processus sédimentaires identifiés. Les informations fournies par les différentes analyses sont alors confrontées et leur convergence permet ou non de décider si l'hypothèse d'une redistribution des vestiges par les processus sédimentaires peut être admise.

Chaque type d'analyse, et notamment la granulométrie, ne donne que des résultats parcellaires ou parfois ambigus et leur interprétation doit s'insérer dans une démarche plus globale. L'application d'une telle démarche permet ainsi de proposer un scénario plausible pour la formation du site, qui rend compte du plus grand nombre d'observations.

En raison des insuffisances actuelles du référentiel sur la composition granulométrique des assemblages lithiques, il semble préférable de restreindre l'analyse aux cas pour lesquels les conditions suivantes sont remplies :

1) la plus petite maille de tamis adoptée est de $2 \mathrm{~mm}$, ce qui permet d'obtenir un spectre très large et des courbes peu sensibles aux facteurs de tri anthropique. Le tamisage doit donc avoir été suffisamment soigneux pour ne pas introduire de biais dans la distribution granulométrique du matériel prélevé.

2) le nombre d'échantillons prélevés sur le site est suffisant pour être représentatif de la distribution granulométrique de l'ensemble du site. Ce nombre peut être faible si aucune aire d'activité particulière n'a été identifiée par la lecture des plans de répartition des objets et si aucune répartition différenciée des catégories d'objets n'apparaît. On estime alors que la distribution du matériel a été homogénéisée sur l'ensemble du site soit par des processus anthropiques (piétinement...), soit par des processus sédimentaires. Cette hypothèse d'homogénéisation ne peut être admise que si tous les échantillons ont une distribution granulométrique similaire. D'une manière pratique, cela revient à effectuer des tests de tamisage en cours de fouille dès qu'une concentration d'objets apparaît et/ou de façon régulière, selon un maillage préétabli.

3) chaque échantillon doit être suffisamment important $(N>100)$ pour limiter les biais statistiques.

\section{6 - EXEMPLES ARCHÉOLOGIQUES : LES SITES DES BOSSES (LOT), DU CASSEUX (LOIR-ET-CHER) ET D'HERMIES (PAS-DE-CALAIS)}

\section{1 - Les Bosses}

Le site des Bosses (Jarry et al. 2001) est localisé à proximité de Cahors, sur une terrasse dominant le cours actuel du Lot d'environ $35 \mathrm{~m}$ (fig. 19). L'étude géologique indique que le niveau archéologique est associé à un pavage graveleux qui recouvre des formations torrentielles déposées par un petit affluent du Lot. Ce niveau est enfoui par des colluvions limoneuses. Le passage d'un contexte érosif associé à la formation du pavage à un contexte d'accumulation sédimentaire à un moment donné du Pléistocène est à mettre en relation avec un soutirage karstique. Ce soutirage et la création consécutive d'une doline ont entraîné la formation d'un piège sédimentaire et ont localement permis au niveau archéologique d'être soustrait à l'érosion. Dans la mesure où le contexte sédimentaire (dépôts torrentiels, pavage) pouvait laisser suspecter une participation importante des processus naturels dans la constitution du niveau archéologique, une analyse granulométrique du matériel récolté a été effectuée pour tenter d'évaluer le rôle de ces processus. 


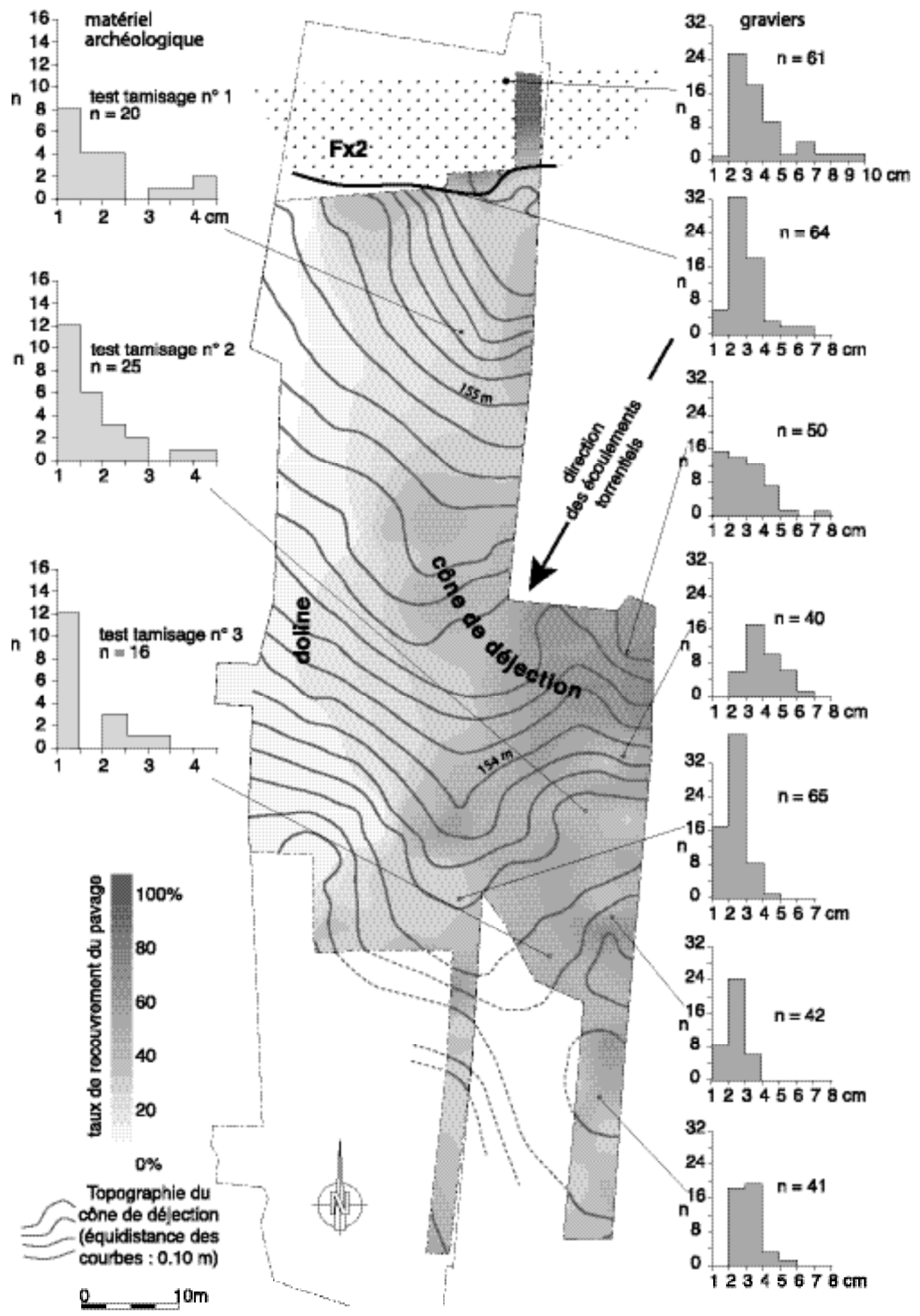

Figure 22 - Granulométrie comparée des graviers torrentiels et du matériel archéologique sur le site des Bosses (Lot), d'après Jarry et al. 2001. Seule est prise en compte la fraction supérieure à $1 \mathrm{~cm}$.

Figure 22 - Compared particle size distribution of the alluvial gravel and the archaeological material of the 'Les Bosses' site (Lot), from Jarry et al. 2001. Only the items $>1 \mathrm{~cm}$ are analysed. 
Les objets archéologiques provenant de trois secteurs ayant fait l'objet d'un tamisage ont été mesurés et la distribution granulométrique peut être comparée à celle des graviers torrentiels. A ce premier stade de l'analyse, seuls les artefacts et les graviers de largeur supérieure à $1 \mathrm{~cm}$ ont été pris en compte. Les résultats sont illustrés sur la figure 22. Ils montrent que :

1) la distribution de la taille des graviers suit approximati vement une loi normale dont le mode est centré sur la classe [2-3 cm]. Elle traduit un tri hydraulique des éléments par les écoulements torrentiels. Une évolution générale s'obsene également de l'amont vers l'aval du cône de déjection : la granulométrie tend à se ressemer et les éléments de taille supérieure à $4 \mathrm{~cm}$ se raréfient vers l'aval. Cette tendance reflète une réduction de la capacité de transport du charriage torrentiel avec la diminution progressive de la pente du cône (Bluck 1964 ; Boothroyd et Nummedal 1978 ; Koulinski 1994) ;

2) la distribution granulométrique du matériel archéologique est différente de celle des matériaux torrentiels. Elle se caractérise par une relative abondance des objets de largeur comprise entre 1 et $2 \mathrm{~cm}$ et par une décroissance du nombre d'éléments vers les grosses tailles (distribution exponentielle décroissante). On en conclut donc que le niveau archéologique n'a pas été affecté par les écoulements torrentiels et que l'occupation du site s'est très probablement produite lorsque le cône de déjection n'était plus actif.
Dans un deuxième stade de l'analyse, la distribution granulométrique d'un échantillon de la série lithique prélevé dans trois secteurs tamisés à $2 \mathrm{~mm}$ a été comparée aux courbes de débitages expérimentaux. Ces courbes comprennent notamment des exemples de débitages sur enclume et de débitages discoïdes unifaciaux sur quartzite comparables au matériel archéologique. Les trois échantillons proviennent de secteurs différents et sont vraisemblablement représentatifs de l'ensemble de l'industrie du site, dans la mesure où aucune aire d'activité particulière ni aucune répartition spatiale différenciée du matériel lithique n'a pu être mise en évidence. La comparaison entre la distribution archéologique et la distribution expérimentale met clairement en évidence un appauvrissement très important de l'assemblage lithique des Bosses en petits et moyens éléments. Seuls 10 objets, soit $16 \%$ du total, ont une largeur inférieure ou égale à $1 \mathrm{~cm}$. L'hypothèse d'un tri granulométrique lié au ruissellement peut être proposée. Le report des valeurs sur le diagramme triangulaire proposé par Lenoble (2005) indique que le déficit dépasse sensiblement celui qu'il a pu obtenir dans le cadre de la plupart de ses expérimentations (fig. 23). Seul un cas s'approche de celui des Bosses : il s'agit d'un assemblage soumis à une longue exposition dans une large rigole. Sur cette base, l'assemblage des Bosses peut être considéré comme un matériel fortement trié au cours d'un transport par le ruissellement. Cette hypothèse est en accord avec d'autres observations :

1) le pourcentage de pièces présentant des signes d'usure est élevé ( $26 \%$ des objets en quartz et $33 \%$ des objets en quartzite sont émoussés) ;

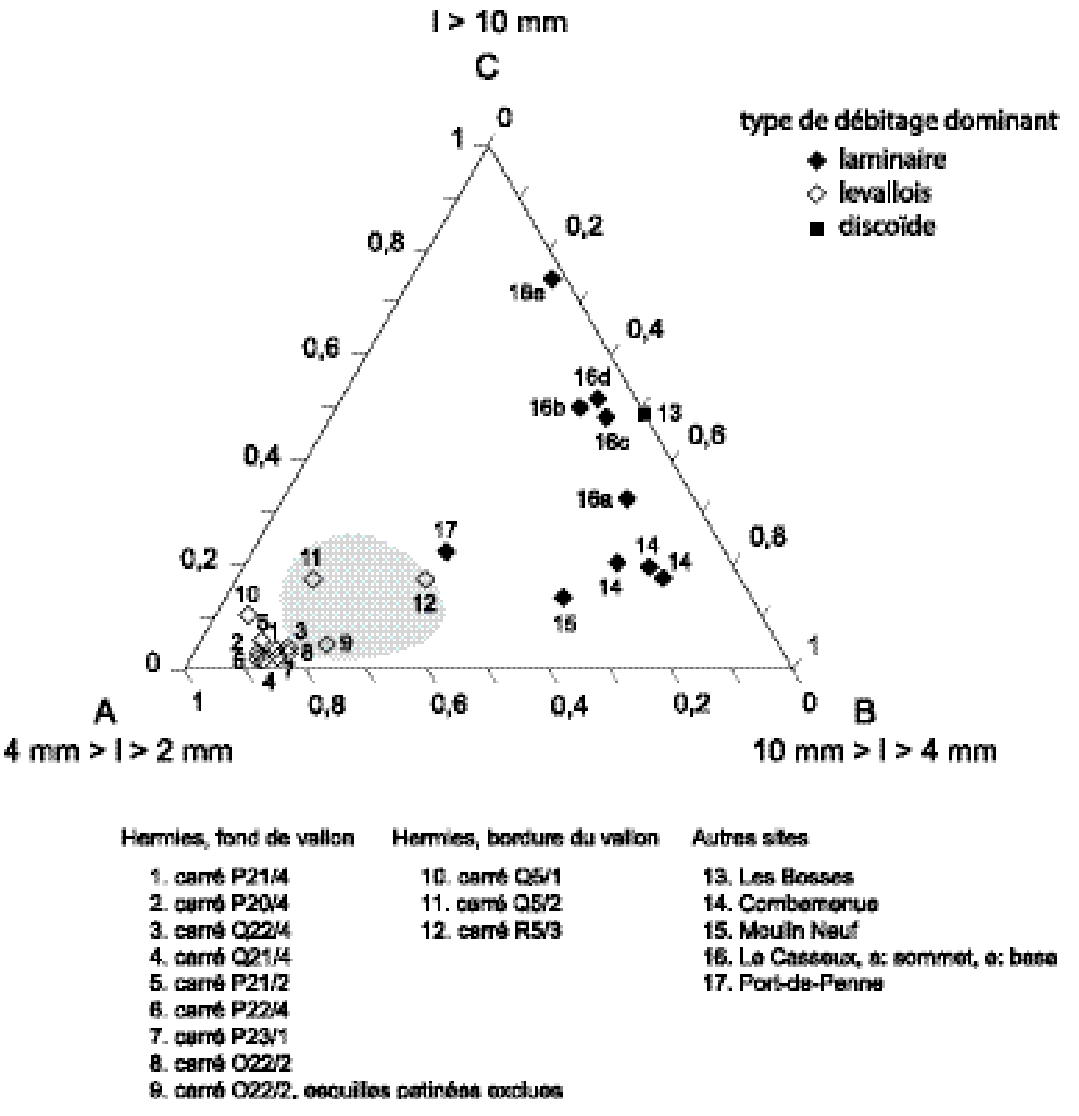

Figure 23 - Composition granulométrique comparée des débitages expérimentaux et de quelques séries archéologiques. Le type de débitage dominant sur chaque site est indiqué par un figuré.

Figure 23 - Compared particle size distribution of experimental debitages and Palaeolithic assemblages. The main debitage type for each site is indicated by a symbol. 
2) le nombre de remontages est faible (le taux de remontage atteint $1,8 \%$ pour le quartzite et $4 \%$ pour le silex, aucun des raccords pour le silex ne concernant plus de 2 pièces à la fois), ce qui suggère qu'une partie significative du matériel a été emportée hors du site par les écoulements ;

3) la répartition en plan des artefacts n'indique pas d'organisation claire du niveau archéologique ; celui-ci s'apparente à une nappe d'objets répartis aléatoirement.

\section{2 - Le Casseux}

Ce site, localisé à proximité de Mareuil-sur-Cher dans le lit majeur du ruisseau du Casseux, a livré des industries du Mésolithique récent et du Magdalénien supérieur (Fourloubey 2004). Le matériel archéologique ne forme pas véritablement de niveaux bien définis mais il est dispersé dans des colluvions recouvrant une nappe d'alluvions sablo-graveleuses. L'étude archéologique a rapidement mis en évidence le caractère remanié des vestiges, en raison du mélange dans toute la séquence et en proportions variables d'un stock de matériel indubitablement mésolithique et d'un stock magdalénien. L'altération prononcée des pièces (lustre, denticulations naturelles sur les tran- chants) ainsi que la fabrique bimodale des objets (présence de deux directions préférentielles disposées à $90^{\circ}$ l'une de l'autre), plaident en faveur d'une redistribution du matériel par des écoulements. L'étude granulométrique effectuée à partir de quelques carrés tests répartis sur toute la hauteur du dépôt vient confirmer cette interprétation. Les points représentatifs des différents lots d'objets s'écartent nettement de la granulométrie connue pour des débitages expérimentaux. Un déficit en éléments de petite ou moyenne taille apparaît clairement (fig. 23) et doit être imputé à l'action des écoulements. Ce déficit est variable : la partie la plus profonde de la couche archéologique (point 16e, fig. 23) est la plus fortement appauvrie, tandis que le matériel issu du décapage sommital (16a) est plus proche de la composition granulométrique attendue. Ces différences peuvent s'interpréter en terme de fluctuations de la compétence des écoulements qui ont affecté le matériel archéologique : la partie inférieure a probablement été redistribuée par des écoulements relativement puissants, comme charge de fond lors des crues du ruisseau, tandis que la partie supérieure n'a été reprise que par des écoulements de faible énergie. Cela suggère une décroissance de l'influence de la dynamique alluviale dans la mise en place du matériel.

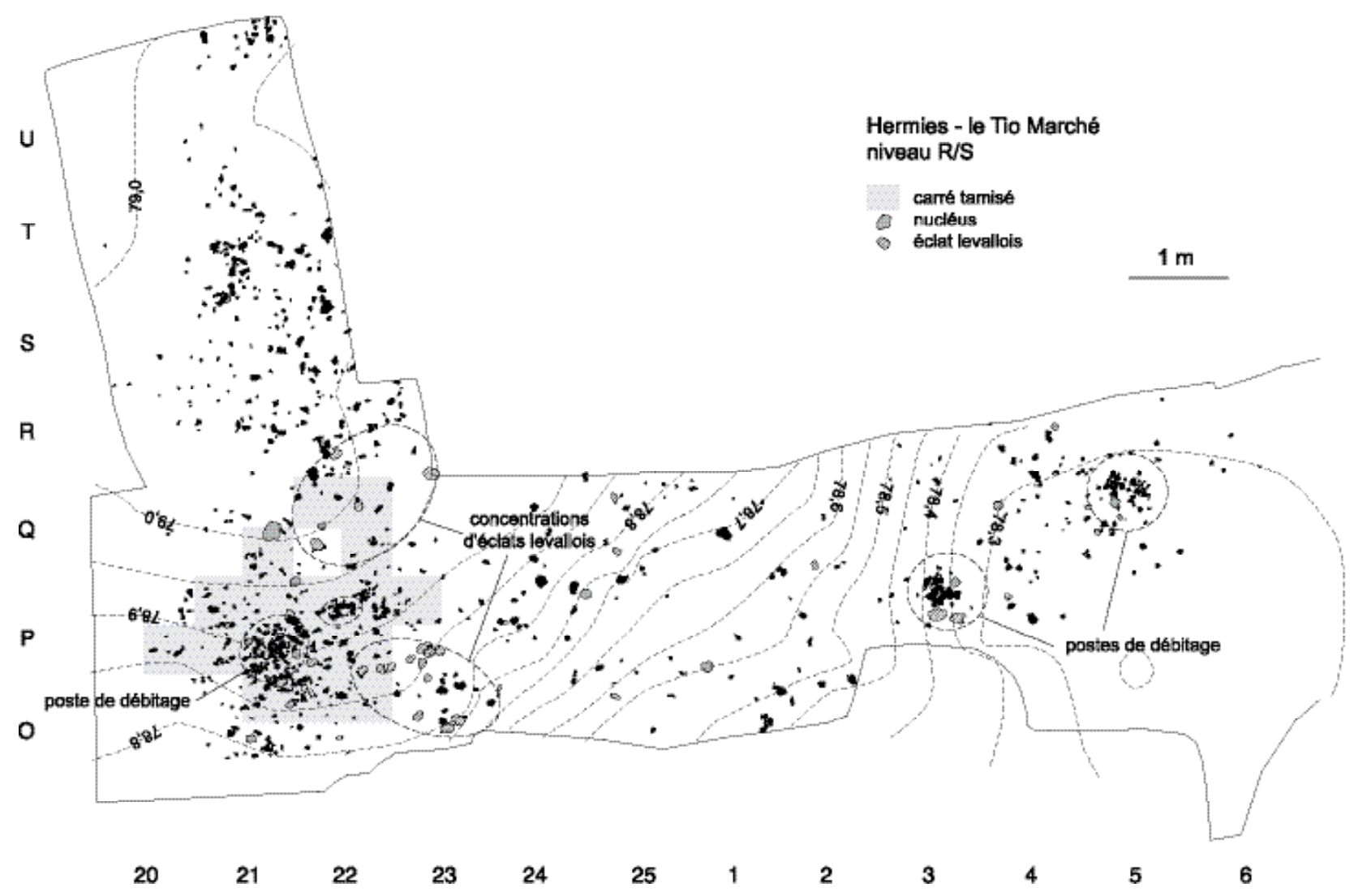

Figure 24 - Plan de répartition des vestiges et localisation des carrés tamisés, Hermies - le Tio Marché (Nord-Pas-de-Calais), niveau $R / S$. L'équidistance des courbes de niveaux est de $5 \mathrm{~cm}$.

Figure 24 - Artefact distribution map and location of the sieved squares, Hermies - Le Tio Marché, level R/S. The contour interval is $5 \mathrm{~cm}$. 
Hermies - Tio Marché

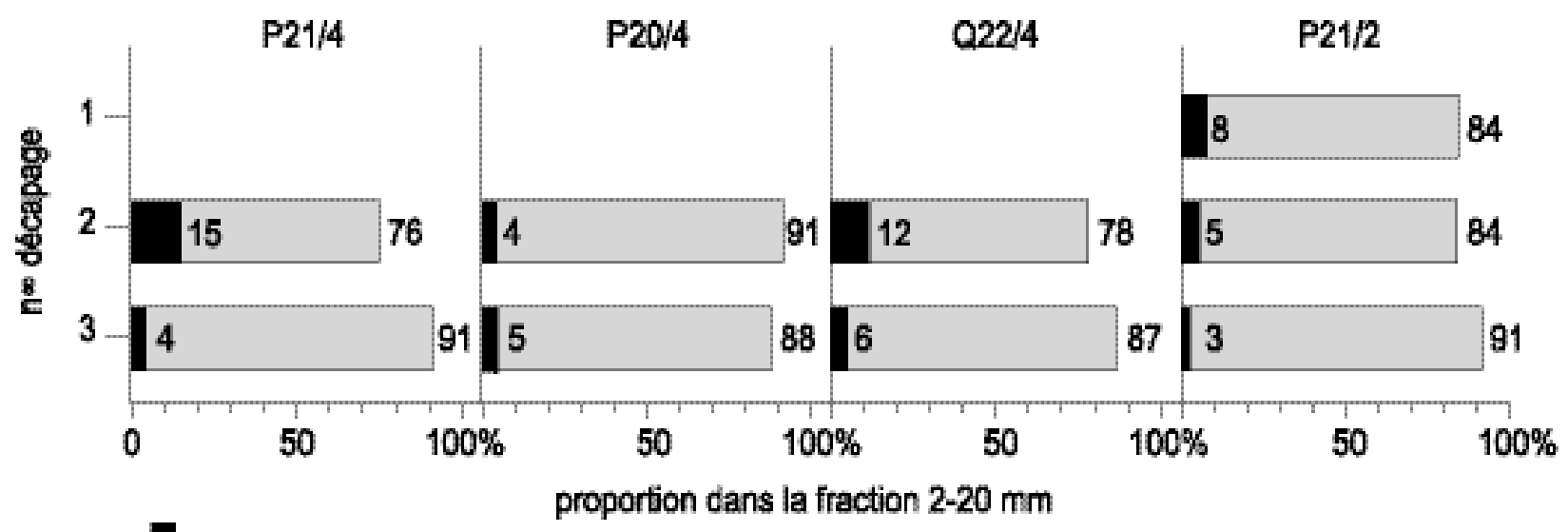

fraction 5-10 mm

fraction $2-4 \mathrm{~mm}$

Figure 25 - Proportion relative des fractions 2-4 mm et 5-10 mm dans la série lithique récoltée dans différents carrés et selon la profondeur, site d'Hermies-Le Tio Marché.

Figure 25 - Proportion of the 2-4 mm and 5-10 mm fractions according to depth at Hermies - Le Tio Marché.

\section{3 - Hermies - Le Tio Marché}

Le site d'Hermies - le Tio Marché est localisé sur les flancs d'un paléovallon incisé dans un plateau loessique à Hemies (Pas-de-Calais) (Vallin et Masson 2005). Ce site a livré une industrie moustérienne essentiellement dominée par le débitage de grands éclats Levallois. Le principal niveau archéologique (R/S) provient d'une couche de loess intercalée dans le complexe de sols de Saint-Acheul, ce qui permet de lui attribuer un âge Pléniglaciaire moyen (stade isotopique 3) (Antoine et al. 2003). Le niveau a été reconnu sur plusieurs dizaines de mètres carrés répartis sur le plateau, les flancs et le fond du petit vallon. II est caractérisé par la présence de concentrations lithiques bien délimitées, qui correspondent à des amas de débitage et à des regroupements d'éclats Levallois utilisés pour des activités de boucherie, séparées par des zones où les objets sont très peu nombreux, voire absents (fig. 24). Le contexte loessique, qui autorise un enfouissement rapide des niveaux archéologiques, et la distribution spatiale différenciée du matériel lithique tendent à indiquer un bon état de préservation du site.

Dans le cadre de cette étude, la granulométrie du matériel récolté en fond de vallon (carrés O-P-Q/20 à 23) et en bordure du vallon (carrés $\mathrm{Q}-\mathrm{R} / 5$ ) a été analysée par tamisage pour la fraction inférieure à $20 \mathrm{~mm}$ et mesure manuelle de la largeur des pièces pour la fraction plus grossière. Les résultats obtenus (fig. 21 ) montrent que la proportion de petits objets (fraction 2-4 mm) est très similaire pour tous les carrés analysés provenant du fond de vallon, qu'ils soient localisés au sein d'un amas de débitage, d'une concentration d'éclats Levallois ou d'une zone pauvre en vestiges. Cette proportion atteint en moyenne 84,5\%, c'est-à-dire qu'elle est sensiblement supérieure à celle des débitages Levallois expérimentaux. Elle suggère donc un enrichissement relatif du niveau archéologique en petits éléments. Cet enrichissement est en revanche moins net pour les carrés $\mathrm{Q}-\mathrm{R} / 5$ en bord de vallon. La distribution verticale des petits éléments, appréhendée à partir du matériel récolté par tamisage des décapages successifs, montre que leur concentration est surtout marquée à la base du niveau archéologique (fig. 25). D'après les observations réalisées en cours de fouille, ce phénomène peut être, au moins pour partie, dû à une fragmentation secondaire sur place du matériel lithique, attribuée au piétinement (Vallin et al. 2005). Des esquillements ont en effet été observés aux points de contact entre les pièces, notamment au sein des amas de débitage. Une telle hypothèse ne permet néanmoins pas de rendre compte du caractère généralisé de l'excès de la fraction 2-4 mm quel que soit le carré considéré et donc, indépendamment de la quantité d'objets de plus grande taille. L'examen de cette fraction au microscope optique en lumière réfléchie fait clairement apparaître qu'elle n'est pas homogène mais qu'elle est constituée d'objets non altérés (fig. 26, 27), d'objets portant un lustre (patine brillante d'origine mécanique ou chimique, fig. 28) et d'objets présentant une patine blanche de dissolution plus ou moins prononcée (fig. 29, 30). Le décompte des esquilles en fonction de la couleur de la patine, effectué à l'œil nu, indique que, selon les carrés analysés, 30 à $50 \%$ d'entre elles ont un aspect blanc laiteux. Moins d'un tiers (10 à $30 \%$ ) ne présente aucune patine, le reste étant dans un état intermédiaire (voile blanchâtre moucheté plus ou moins marqué). Si l'on exclut les pièces les plus altérées (patine blanche), la composition granulo- 

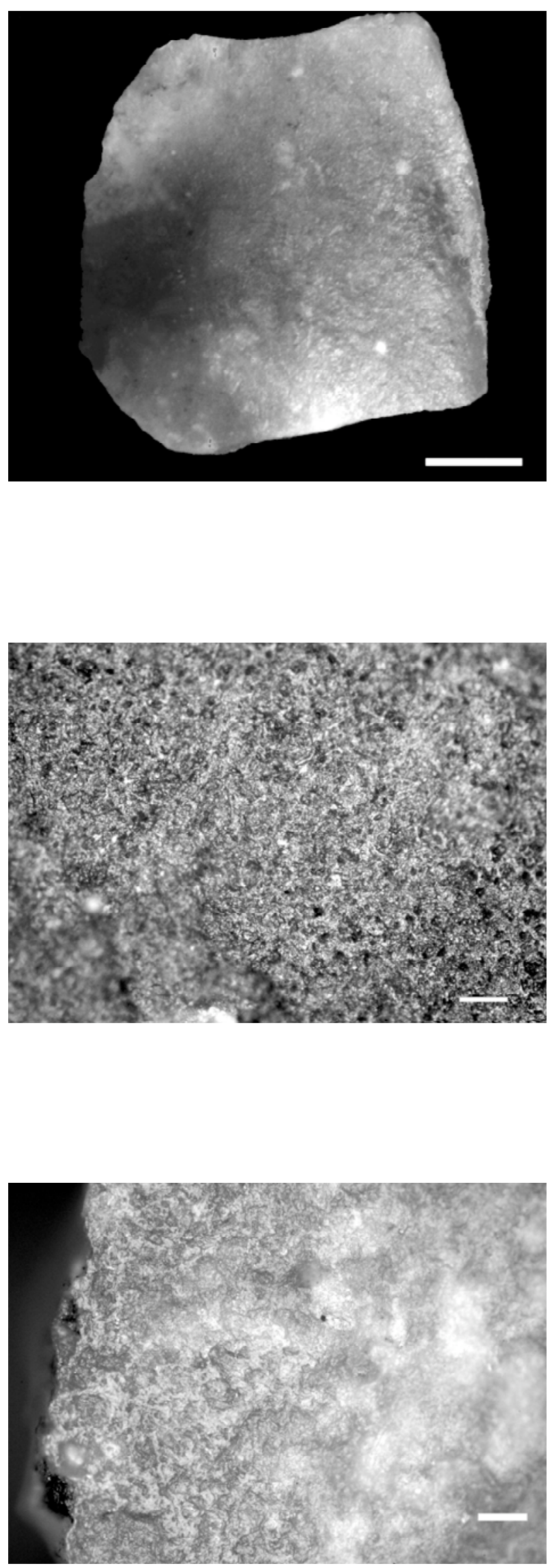

Figure 26 - Silex gris foncé non ou faiblement altéré. L'échelle fait 0,5 mm. Hermies - Le Tio Marché, microscopie optique en lumière réfléchie.

Figure 26 - Unweathered dark grey flint fragments. The scale is $0.5 \mathrm{~mm}$. Hermies - Le Tio Marché, reflected light.

Figure 27 - Etat de surface d'une esquille de silex gris foncé non altéré. L'échelle fait $50 \mathrm{~mm}$ (grossissement x200). Hermies - Le Tio Marché, microscopie optique en lumière réfléchie.

Figure 27 - Close-up of an unweathered dark grey flint fragment. The scale is $50 \mathrm{~mm}$. Hermies - Le Tio Marché, reflected light.

Figure 28 - Patine brillante (lustre) affectant les reliefs dans la partie gauche de la photo et patine blanche à droite. L'échelle fait $50 \mathrm{~mm}$ (grossissement x200). Hermies - Le Tio Marché, microscopie optique en lumière réfléchie.

Figure 28 - Shiny patina on reliefs on the left part of the photo and spots of white patina on the right. The scale is $50 \mathrm{~mm}$. Hermies - Le Tio Marché, reflected light. 

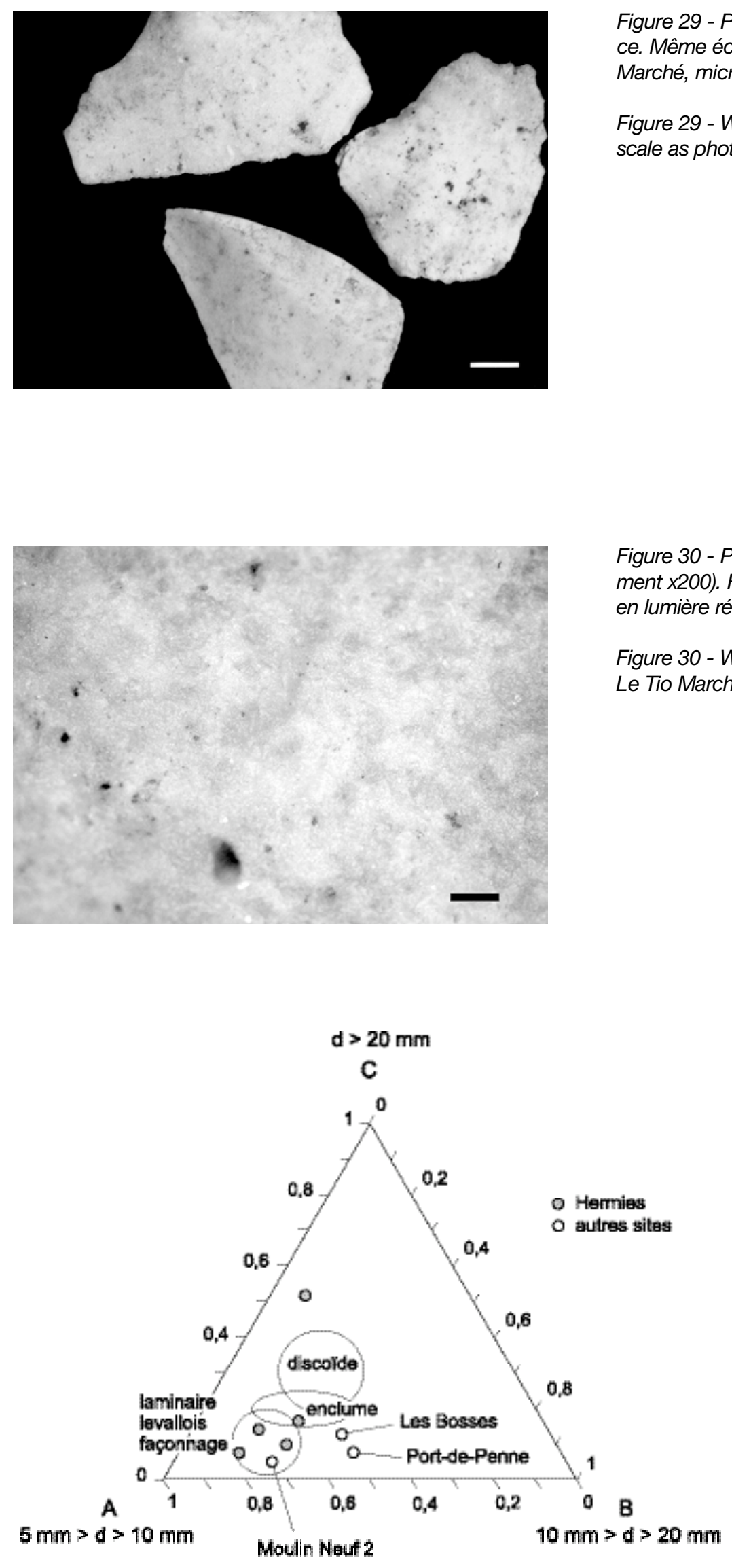

Figure 29 - Patine blanche couvrant l'ensemble de la surface. Même échelle que la photo 5. Hermies - Le Tio Marché, microscopie optique en lumière réfléchie.

Figure 29 - White patina covering the whole surface. Same scale as photo 5. Hermies - Le Tio Marché, reflected light.
Figure 30 - Patine blanche. L'échelle fait $50 \mathrm{~mm}$ (grossissement x200). Hermies - Le Tio Marché, microscopie optique en lumière réfléchie.

Figure 30 - White patina. The scale is $50 \mathrm{~mm}$. Hermies Le Tio Marché, reflected light.
Figure 31 - Granulométrie de la fraction > 5 $\mathrm{mm}$ provenant de différents carrés d'Hermies - Le Tio Marché. L'aire correspondant aux débitages expérimentaux et la composition de quelques autres sites évoqués plus haut sont également indiquées.

Figure 31 - Size distribution of the fraction > $5 \mathrm{~mm}$ in some squares at Hermies - Le Tio Marché. The area of experimental debitages and the composition of other already-mentioned Palaeolithic sites are indicated. 
métrique devient similaire à celle des débitages expérimentaux. L'hypothèse d'une redistribution partielle d'occupations " anciennes " déjà patinées situées à l'amont et d'une accumulation par le ruissellement en fond de vallon semble donc pouvoir être retenue. Cette redistribution concerne approximativement un tiers de la fraction 2-4 $\mathrm{mm}$. L'association d'un tri granulométrique et de structures archéologiques apparemment bien préservées reflète probablement un stade initial de dégradation du site, en liaison avec une exposition de relativement courte durée aux processus sédimentaires superficiels. Ainsi, lorsque l'on ne prend pas en compte la fraction $<5 \mathrm{~mm}$, qui est la plus mobile et qui est susceptible d'être redistribuée rapidement après l'abandon du site par les paléolithiques, la composition granulométrique de la plupart des carrés analysés entre dans le champ des débitages expérimentaux (fig. 31). Seul se distingue le carré $\mathrm{P} 21 / 2$, qui correspond à un petit amas en périphérie d'un poste de débitage plus important et montre une surreprésentation des pièces de largeur (l) supérieure à $28 \mathrm{~mm}(\mathrm{~d}=20 \mathrm{~mm})$. Ce phénomène pourrait refléter un tri d'origine anthropique. L'analyse technologique de l'amas indique qu'il comprend essentiellement deux segments de séquence de débitage Levallois. L'une des séquences (46 objets remontés) correspond aux premières étapes de mise en forme du nucléus (décorticage, mise en place des plans de frappe et aménagement des convexités de la surface Levallois), l'autre (17 objets) à une phase de plein débitage (repréparation du plan de frappe et de la surface Levallois, puis débitage d'un éclat préférentiel). Cette analyse permet de rejeter l'hypothèse d'une concentration d'objets sélectionnés ; la surreprésentation de la fraction grossière pourrait être liée soit au faible effectif de la série, soit aux caractéristiques mêmes du débitage.

\section{7 - CONCLUSION}

A l'issue de ce travail, l'analyse granulométrique des assemblages lithiques apparaît d'ores et déjà comme un outil efficace au service des études de taphonomie archéologique. Le fondement de cette analyse repose sur la comparaison entre la composition de la fraction supérieure à $2 \mathrm{~mm}$ d'un assemblage archéologique et celle de débitages expérimentaux. Les écarts importants de composition indiquent la présence d'un tri granulométrique. Si le référentiel expérimental semble déjà suffisamment étoffé pour être représentatif de la variabilité induite par le type de débitage ou celui de la matière première et par conséquent, pour que l'identification d'un tri granulométrique ne présente pas d'ambiguïté, l'interprétation de l'origine de ce tri, i.e. naturel ou anthropique, reste encore délicate. Cela tient essentiellement au manque de données concernant la variabilité granulométrique liée à la spatialisation des activités humaines sur un site. Des progrès significatifs peuvent donc être attendus de l'étude de sites bien préservés, dans lesquels la composition du matériel provenant des différentes zones d'activité pourra être documentée. Malgré tout, dans l'état actuel des connaissances, l'association d'un tri granulométrique et d'autres indices témoignant de l'action du ruissellement ou d'une dynamique alluviale (structures sédimentaires, abrasion des pièces...), constitue un argument solide en faveur de l'hypothèse d'une dégradation notable du site par les écoulements.

\section{Remerciements}

Ce travail a été effectué dans le cadre de l'ACR intitulé " Taphonomie des assemblages paléolithiques moyen du Nord de la France " dirigé par L. Vallin. Sont remerciées les différentes personnes qui ont taillé les débitages expérimentaux : B. Aubry, L. Bourguignon, M. Brenet, A. Delagnes, P. Fouéré, J. Pelegrin et V. Mourre. Les responsables des sites archéologiques analysés (C. Fourloubey, M. Jarry, M. Lenoir) et les relecteurs des premières versions de l'article (J. Pelegrin, J.P. Texier et J. Tixier) sont vivement remerciés.

\section{BIBLIOGRAPHIE}

ANTOINE P., BAHAIN J.J., DEBENHAM N., FRECHEN M., GAUTHIER A., HATTE C., LIMONDIN-LOZOUET N., LOCHT J.L., RAYMOND P. et D.D. ROUSSEAU 2003 Nouvelles données sur le Pléistocène du nord du Bassin Parisien : les séquences loessiques de Villiers-Adam (Val d'Oise, France). Quaternaire, 14 (4) : 219-236

BERTRAN P. et TEXIER J.-P. 1994 - Structure sédimentaire d'un cône de flot de débris (Vars, Alpes françaises méridionales). Permafrost and Periglacial Processes, 5, p. 155-170.

BERTRAN P. sous presse - Genèse et diagenèse du site archéologique. In : Les Bosses à Lamadelaine (Lot, France) : un site paléolithique moyen ancien du Lot, M. Jarry (dir.), Mémoire de la Société Préhistorique Française.

BLUCK B.J. 1964 - Sedimentation of an alluvial fan in southemNevada. Joumal of Sedimentary Petrology, 34 (2), p. 395-400.

BOOTHROYD J.C. et NUMMEDAL D. 1978 - Proglacial braided outwash: a model for humid alluvial-fan deposits. Fluvial sedimentology, Canadian Society of Petroleum Geologists, memoir 5, p. 641-668.

COLCUTT S.N., BARTON N.R.E. et BERGMAN C.A. 1990 - Reffiting in context : a taphonomic case study from a late Upper Palaeolithic site in sands on Hengistburry Head, Dorset, Great Britain. The Big Puzzle, E. Ciesla, S. Eickhoff, N. Arts et D. Winter (eds.), Bonn, p. 219-235.

DETRAIN L., CHAUVIERE F.-X., DIOT M.-F., KERVAZO B., MARTIN H. et O'YI W. 1996 - Camping du Saut, Port-dePenne, Penne d'Agenais, Lot-et-Garonne. Document Final de Synthèse, AFAN Grand-Sud-Ouest, Pessac, 90 p.

FOURLOUBEY C. 2004 - Les occupations magdalénienne et néolithique du "Casseux" à Mareuil-sur-Cher (Loir-etCher). Site A85 $n^{\circ} 42$. Notice intermédiaire de diagnostic 
archéologique. Rapport non publié, Service Régional de l'Archéologie, Orléans, 34 p.

FROSTICK L.E. et REID I. 1983 - Taphonomic signifiance of sub-aerial transport of vertebrate fossils on steed semiarid slopes. Lethia, 16, p. 157-164

JARRY M., BERNARD F., BERTRAN P., CHALARD P., COLONGE D., DEBENHAM N., DIOT M.-F., LELOUVIER L.-A., MOURRE V., TIXIER C. et TURQ A. 2001 - Les Bosses 1. Un gisement paléolithique moyen antérieur à l'avant-demier interglaciaire sur la terrasse moyenne du Lot. Document Final de Synthèse, INRAP Grand-SudOuest, Pessac, 248 p.

HANSEN P.V. et MADSEN B.O. 1983 - Flint axe manufacture in the Neolithic. An experimental investigation of a flint axe manufacture at Hastrup Vaenget, East Zealand. Joumal of Danish Archaeology, 2, p. 43-59.

HJULSTRÖM F. 1939 - Transportation of detritus by moving water. Recent marine sediments, P.D. Trash (ed), American association of Petrology and Geology, p. 5-31.

KLUSKENS S.L. 1990 - Orientation and density analysis of stone artifacts at Combe Capelle bas, Périgord, France. Master Thesis, Université de Pennsylvanie, Philadelphie, $118 \mathrm{p}$.

KOULINSKI V. 1994 - Etude de la formation d'un lit torrentiel. Etudes du CEMAGREF, Equipements pour l'eau et l'environnement n¹5, CEMAGREF, Grenoble, 538 p.

LENOBLE A. 2005 - Ruissellement et formation des sites préhistoriques : référentiel actualiste et exemples d'application au fossile. British Archaeological Report Intemational Series, Oxford, $\mathrm{n}^{\circ} 1363,212 \mathrm{p}$.

LENOIR M. 1983 - Le Paléolithique des basses vallées de la Dordogne et de la Garonne. Thèse d'état, université de Bordeaux 1, $702 \mathrm{p}$.

PATTERSON L.W. 1990 - Characteristics of bifacial-reduction flake-size distribution. American Antiquity, 55 (3), p. 550-558.

PETRAGLIA M.D. et NASH D.T. 1987 - The impact of fluvial processes on experimental sites. Natural formation processes and the archaeological record. D.T. Nash et M.D. Petraglia (eds.), B.A.R. International Series, 352, Oxford, p. 108-130
PIGEOT N. (dir.) 2004 - Les derniers Magdaléniens d'Etiolles. Perspectives culturelles et paléohistoriques. XXXVII ${ }^{\mathrm{e}}$ supplément à Gallia Préhistoire, CNRS, Paris, 351 p.

RAMEZ P. 1995 - Erosion et transport solide en rivière. Tome 1 : Guide pour la compréhension des phénomènes. Cemagref, Antony, $130 \mathrm{p}$.

REINECK H.E. et SINGH I.B. 1980 - Depositional Sedimentary Environments. Second edition, SpringerVerlag, Berlin, $551 \mathrm{p}$.

SCHICK K.D. 1986 - Stone Age sites in the making. Experiments in the formation and transformation of archaeological occurrences. BAR International Series 319, $313 \mathrm{p}$.

STAHLE D.W. et DUNN J.E. 1982 - An analysis and application of the size distribution of waste flakes from the manufacture of bifacial stone tools. World Archaeology, 14 (1), p. 84-97.

TIXIER J., INIZAN M. et ROCHE H. 1980 - Préhistoirede la pierre taillée : 1 - Te rminologie et technologie. Cercle de Recherches et d'Etudes Préhistoriques, Valbonne, 120 p.

VALLIN L. et MASSON B. 2005 - Le gisement moustérien d'Hermies - le Tio Marché. Rapport de synthèse sur les fouilles programmées 1997-2003. Service Régional de l'Archéologie du Nord-Pas-de-Calais, Villeneuve d'Ascq, $167 \mathrm{p}$.

VALLIN L., ANTOINE P., AUBRY B., CASPAR J.P., CLIQUET D., DELAGNES A., DEPAEPE P., GUILLEMET G., LAIGNEL B., LOCHT J.L., MASSON B., OZOUF J.-C. et SWINNEN C. 2005 - Taphonomie des assemblages lithiques du Paléolithique moyen en contexte périglaciaire : approches expérimentale et archéologique à partir de sites du nord-ouest européen. Rapport non publié, Service Régional de l'Archéologie du Nord-Pas-de-Calais, Villeneuve d'Ascq, $115 \mathrm{p}$.

VAN NEST J. 2002 - The good earthworm : how natural processes preserve upland Archaic archaeological sites of Westemlllinois, U.S.A. Geoarchaeology, 17 (1), p. 53-90. 
